\title{
The importance of surface pressure changes in the response of the atmosphere to zonally-symmetric thermal and mechanical forcing
}

Article

Published Version

Haynes, P. H. and Shepherd, T. G. (1989) The importance of surface pressure changes in the response of the atmosphere to zonally-symmetric thermal and mechanical forcing. Quarterly Journal of the Royal Meteorological Society, 115 (490). pp. 1181-1208. ISSN 1477-870X doi:

https://doi.org/10.1002/qj.49711549002 Available at https://centaur.reading.ac.uk/32988/

It is advisable to refer to the publisher's version if you intend to cite from the work. See Guidance on citing.

Published version at: http://dx.doi.org/10.1002/qj.49711549002

To link to this article DOI: http://dx.doi.org/10.1002/qj.49711549002

Publisher: Royal Meteorological Society

All outputs in CentAUR are protected by Intellectual Property Rights law, including copyright law. Copyright and IPR is retained by the creators or other copyright holders. Terms and conditions for use of this material are defined in the End User Agreement. 


\section{www.reading.ac.uk/centaur}

\section{CentAUR}

Central Archive at the University of Reading

Reading's research outputs online 
OF THE

\title{
ROYAL METEOROLOGICAL
}

SOCIETY

\begin{tabular}{lll}
\hline \hline Vol. 115 & OCTOBER 1989 & No. 490 \\
\hline \hline
\end{tabular}

Q. J. R. Meteorol. Soc. (1989), 115, pp. 1181-1208

$551.511 .3: 551.543$

\section{The importance of surface pressure changes in the response of the atmosphere to zonally-symmetric thermal and mechanical forcing}

\author{
By P. H. HAYNES and T. G. SHEPHERD* \\ Department of Applied Mathematics and Theoretical Physics, University of Cambridge
}

(Received 21 July 1988; revised 3 April 1989)

SUMMARY

The classical problem of the response of a balanced, axisymmetric vortex to thermal and mechanical forcing is re-examined, paying special attention to the lower boundary condition. The correct condition is $D \Phi / D t=0$, where $\Phi$ is the geopotential and $D / D t$ the material derivative, which explicitly accounts for a mass redistribution as part of the mean-flow response. This redistribution is neglected when using the boundary condition $D p / D t=0$, which has conventionally been applied in this problem. It is shown that applying the incorrect boundary condition, and thereby ignoring the surface pressure change, leads to a zonal wind acceleration $\partial \bar{u} / \partial t$ that is too strong, especially near the surface. The effect is significant for planetary-scale forcing even when applied at tropopause level.

A comparison is made between the mean-flow evolution in a baroclinic life-cycle, as simulated in a fully nonlinear, primitive-equation model, and that predicted by using the simulated eddy fluxes in the zonally. symmetric response problem. Use of the correct lower boundary condition is shown to lead to improved agreement.

\section{INTRODUCTION}

In the classical theory of a balanced, axisymmetric vortex (Eliassen 1951), the application of a zonally-symmetric thermal or mechanical force induces a response that acts to keep the vortex in a state of balance. The response consists of both an adjustment of the wind and temperature fields of the vortex, as well as an induced circulation in the meridional plane. This viewpoint has proved to be of great utility in understanding the effects of eddy transports and of friction on the zonally-symmetric circulation (Eliassen 1951; Kuo 1956; Dickinson 1969; Andrews and McIntyre 1976; Pfeffer 1981, 1987; Crawford and Sasamori 1981; Plumb 1982; Hayashi 1985; Palmer et al. 1986), including the important question of the 'maintenance of the westerlies'.

The mathematical structure of the quasi-geostrophic response problem is that of a Poisson equation, namely of the form $\mathscr{L}(\phi)=\mathscr{F}$, where $\phi$ is a mean-flow variable (for example, the zonal wind acceleration $\partial \bar{u} / \partial t), \mathscr{F}$ is a forcing term, and $\mathscr{L}$ is an elliptic operator. For given $\mathscr{F}$, the operator $\mathscr{L}$ must be inverted to find the response $\phi$. In solving such an inversion problem, appropriate boundary conditions must be imposed. In the meteorological context one usually considers a semi-infinite atmosphere, either in a zonally-symmetric channel or on the sphere. While the upper and lateral boundary conditions are then straightforward (no vertical mass flux, and no meridional flow, respectively), the lower boundary condition is more problematical. The correct condition

* Present address: Department of Physics, University of Toronto, Canada 
is that there be no flow across the lower boundary. If the boundary is a (horizontal) geopotential surface, then in geometrical coordinates the vertical velocity must vanish there, equivalently $D \Phi / D t=0$ where $\Phi$ is the geopotential and $D / D t$ the material derivative. It does not follow that the vertical velocity in pressure coordinates, $\omega=D p / D t$, or that in log-pressure coordinates, $w=D z / D t=-H \omega / p$ need vanish, even in the absence of topography.

Nonetheless, for reasons of computational simplicity, or otherwise, it has been conventional to solve the inversion problem under the assumption that the lower boundary be a streamline for the induced meridional circulation in pressure or log-pressure coordinates, equivalently that $\bar{\omega}$ or $\bar{w}$ must vanish there. The defence of this procedure has presumably been that the effect of a surface pressure tendency would be small. The effect is frequently neglected in quasi-geostrophic theory, but this cannot be justified on the basis of small Rossby number alone. Rather, it requires an additional quasi-Boussinesq assumption that the density tendency $\partial \rho / \partial t$ can be neglected in the (geometriccoordinate) continuity equation (see e.g. White 1977). This assumption is invalid whenever the rotational Mach number, defined by (2.19) below, is order unity or greater, which is certainly the case for motion of sufficiently large horizontal scale.

Indeed, it is the purpose of this paper to point out that, in many instances, allowing for a surface pressure tendency as part of the inversion problem can substantially change the nature of the zonal-mean response at leading order. While the effects may be expected to be small for forcing located well above the tropopause (because the high static stability implies that the distance to the ground is effectively large), and for small horizontal scales (because the corresponding vertical scales are much less than one scale height), it will be seen that for planetary-scale forcing within the troposphere, even at the tropopause level itself, the effects can be very significant. One implication of this result is that one is no longer justified in viewing the meridional circulation as acting to redistribute only the relative angular momentum, as it must do when the circulation forms a closed meridional cell so that there is no net transport of planetary angular momentum across any latitude circle.

Whilst it is clear that, in a time-averaged problem, the surface pressure tendency must vanish, the tendencies in zonal velocity and temperature are themselves subject to the same constraint. This does not prevent one from using the time-dependent problem to gain insight into the contribution of various forcings to the maintenance of the seasonal mean state. But it is essential that this problem be posed self-consistently. Just as the response in the tendencies of zonal velocity and temperature to the observed eddy forcing must, in the time mean, be cancelled by the response induced by frictional and other diabatic effects, so too should the response in surface pressure tendency. It is clearly inconsistent to assume that the surface pressure response vanishes separately for each component of the forcing.

The plan of the paper is as follows. In section 2 the inversion problem on the sphere (including the surface pressure tendency in the lower boundary condition) is defined mathematically, and a solution algorithm described which follows Plumb (1982) in using a Hough-function decomposition of the latitudinal structure and allows for the effects of variable Coriolis parameter. This provides a set of ordinary differential equations which may be solved to obtain the vertical dependence of the Hough-function coefficients. In the process, the conditions under which $\omega=0$ is a good approximation to the lower boundary condition are clarified. In section 3 some important effects arising from surface pressure changes are presented and highlighted by a set of idealized numerical and analytical calculations. Then in section 4 a comparison is made between the mean-flow evolution in a baroclinic life-cycle, as simulated in a fully nonlinear, primitive-equation 
model, and that predicted by using the simulated eddy fluxes in the quasi-geostrophic inversion problem. This includes explicit consideration of the ageostrophic parts of the eddy forcing terms. Some implications for diagnostic studies of the general circulation are discussed in section 5 .

\section{MATHEMATICAL FORMULATION}

The system under consideration is zonally-symmetric quasi-geostrophic flow driven by thermal and mechanical forcing, for which the governing equations may be written

$$
\begin{gathered}
\partial u / \partial t-2 \Omega \mu v=F \\
2 \Omega \mu u=-a^{-1}\left(1-\mu^{2}\right)^{1 / 2} \partial \Phi / \partial \mu \\
\partial \Phi / \partial p=-R T / p \\
\partial T / \partial t-\Gamma \omega=Q \\
a^{-1} \frac{\partial}{\partial \mu}\left\{\left(1-\mu^{2}\right)^{1 / 2} v\right\}+\frac{\partial \omega}{\partial p}=0 .
\end{gathered}
$$

In the above, $p$ is pressure, $t$ time, $\mu=\sin$ (latitude), $u$ and $v$ are the zonal and meridional velocities, $\omega=D p / D t, T$ is temperature, $\Phi$ the geopotential, $a$ and $\Omega$ the radius and rotation rate of the earth, respectively, $R$ the gas constant, $\Gamma=-T \partial \ln \theta / \partial p$ a static stability parameter (where $\theta$ is the potential temperature) which is assumed to be independent of $\mu$, and $F$ and $Q$ respectively the mechanical and thermal forcing terms (possibly including eddy-flux divergences). All dependent variables and parameters are zonally symmetric (and so overbars will not be used). Note that under the quasigeostrophic approximation the mean advection terms and the horizontal variation of static stability are neglected in (2.1), (2.2) and (2.4), which is equivalent to a linearization about a state of rest, with the additional requirement that the flow remain in geostrophic balance. The thermal wind equation is obtained by eliminating $\Phi$ from (2.2) and (2.3), and takes the form

$$
2 \Omega \mu \frac{\partial u}{\partial p}=a^{-1}\left(1-\mu^{2}\right)^{1 / 2} \frac{R}{p} \frac{\partial T}{\partial \mu} .
$$

Boundary conditions are taken to be $v=0$ at $\mu=-1$ and $\mu=1$ (the poles), $\omega=0$ at $p=0$, and $D \Phi / D t=0$ at the lower surface $p=p_{\mathrm{s}}$. Within the quasi-geostrophic approximation this last condition may be applied at a constant pressure $p_{0}$; this is because although the changes in surface pressure $p_{\mathrm{s}}$ are dynamically significant, they are nevertheless small when compared to $p_{\mathrm{o}}$. The condition may be written as

$$
\partial \Phi / \partial t+\omega \partial \Phi_{\mathrm{o}} / \partial p=0 \text { at } \quad p=p_{\mathrm{o}}
$$

where $\Phi_{\mathrm{o}}$ is the geopotential in the resting basic state. While (2.7) is the proper lower boundary condition for the linearized problem (in the absence of topography), it is conventionally - and in some cases inconsistently-approximated by $\omega=0$ (as discussed in the introduction). Here the full condition (2.7) is of course used.

The above set of equations describes the balanced response of the zonally-symmetric flow to prescribed forcing. Its physical relevance depends on the zonal flow being stable to symmetric disturbances (Eliassen 1951; Kuo 1956), which is generally the case for the large-scale circulation. The response comes partly through time tendencies of the zonal wind and temperature fields, and partly through an induced circulation in the meridional plane. It follows in general that there is a time tendency in the geopotential at constant 
pressure $p_{0}$. With the lower boundary condition (2.7), but not with $\omega=0$, this tendency will be consistently reflected in a net meridional mass flux.

The equations may be used to derive a single equation in any one of the variables $\partial u / \partial t, \partial T / \partial t, v$ or $\omega$, with independent variables $p$ and $\mu$. For example, following Plumb (1982) one may eliminate all but $\omega$ to obtain

$$
\frac{\partial}{\partial \mu}\left\{\frac{\left(1-\mu^{2}\right)}{\mu^{2}} \frac{\partial \omega}{\partial \mu}\right\}+\frac{4 \Omega^{2} a^{2} p}{R \Gamma} \frac{\partial^{2} \omega}{\partial p^{2}}=\frac{2 \Omega a p}{R \Gamma} \frac{\partial}{\partial \mu}\left\{\frac{\left(1-\mu^{2}\right)^{1 / 2}}{\mu} \frac{\partial F}{\partial p}\right\}-\Gamma^{-1} \frac{\partial}{\partial \mu}\left\{\frac{\left(1-\mu^{2}\right)}{\mu^{2}} \frac{\partial Q}{\partial \mu}\right\}
$$

corresponding to Plumb's equation (2.10), which is an elliptic equation for $\Gamma>0$ and may therefore be 'inverted' to find $\omega$ from $F$ and $Q$. (Note that the $Q$ used here corresponds to Plumb's $Q_{*}$.) The boundary condition (2.7) may be expressed in terms of $\omega$ alone, namely

$$
\rho_{\mathrm{o}}^{-1} \frac{\partial}{\partial \mu}\left\{\frac{\left(1-\mu^{2}\right)}{\mu^{2}} \frac{\partial \omega}{\partial \mu}\right\}-4 \Omega^{2} a^{2} \frac{\partial \omega}{\partial p}=-2 \Omega a \frac{\partial}{\partial \mu}\left\{\frac{\left(1-\mu^{2}\right)^{1 / 2}}{\mu} F\right\} \quad \text { at } \quad p=p_{\mathrm{o}}
$$

noting that $\partial \Phi_{0} / \partial p=-\rho_{0}^{-1}$, with $\rho_{0}$ the basic-state density at $p=p_{0}$. It will become clear in section 3 , once the nature of the solutions has been considered, that using (2.9) in place of $\omega=0$ does not alter the uniqueness property of the solutions, and in particular does not allow non-trivial free solutions. Once $\omega$ is determined the other response variables may then be found from the original set of equations.

Again following Plumb (1982), the latitudinal structure of the dependent variables is expressed in terms of either zero-frequency, zonally-symmetric Hough functions $\Theta_{n}(\mu)$, or the associated functions $B_{n}(\mu)$ which are related to $\Theta_{n}(\mu)$ by

$$
\Theta_{n}(\mu)=\frac{d}{d \mu}\left\{B_{n}(\mu)\left(1-\mu^{2}\right)^{1 / 2}\right\}
$$

To wit, one introduces expansions as follows:

$$
\left.\begin{array}{rlrl}
\omega(p, \mu) & =\sum_{n} \omega_{n}(p) \Theta_{n}(\mu) ; & v(p, \mu) & =\sum_{n} v_{n}(p) B_{n}(\mu) ; \\
\frac{\partial T}{\partial t}(p, \mu) & =\sum_{n} T_{n}(p) \Theta_{n}(\mu) ; & \frac{\partial u}{\partial t}(p, \mu) & =\sum_{n} u_{n}(p) \mu B_{n}(\mu) ; \\
Q(p, \mu) & =\sum_{n} Q_{n}(p) \Theta_{n}(\mu) ; & F(p, \mu) & =\sum_{n} F_{n}(p) \mu B_{n}(\mu) .
\end{array}\right\}
$$

Note that the boundary condition for $v$ at the poles is satisfied automatically by virtue of the fact that $B_{n}( \pm 1)=0$, which is required if $B_{n}(\mu)$ is to be regular. Substituting these expansions into (2.8), and using the fact that the Hough functions satisfy the eigenvalue equation

$$
\frac{d}{d \mu}\left\{\frac{\left(1-\mu^{2}\right)}{\mu^{2}} \frac{d \Theta_{n}}{d \mu}\right\}-\epsilon_{n} \Theta_{n}=0
$$

together with $(2.10)$, one obtains the following set of ordinary differential equations in $p$ for the expansion coefficients $\omega_{n}$ :

$$
\frac{4 \Omega^{2} a^{2} p}{R \Gamma} \frac{d^{2} \omega_{n}}{d p^{2}}+\epsilon_{n} \omega_{n}=\frac{2 \Omega a p}{R \Gamma} \frac{d F_{n}}{d p}-\Gamma^{-1} \epsilon_{n} Q_{n}
$$


corresponding to Plumb's (5.8). (Note that the Hough-mode eigenvalues $\epsilon_{n}$ given by Plumb (1982, Table 1) are incorrect by factors of $15 \%$ or so. The correct values are given by Longuet-Higgins (1968, Table 10).) The set of equations (2.13) must be solved subject to the boundary conditions

$$
\begin{gathered}
\omega_{n}=0 \quad \text { at } \quad p=0 \\
\rho_{\mathrm{o}}^{-1} \epsilon_{n} \omega_{n}-4 \Omega^{2} a^{2}\left(d \omega_{n} / d p\right)=-2 \Omega a F_{n} \quad \text { at } p=p_{\mathrm{o}}
\end{gathered}
$$

the last equation arising from substitution of the expansions (2.11) into (2.9). (These differ from Plumb's boundary conditions (6.2) and (6.3).) The problem defined by (2.13) and (2.14) can then be written in finite-difference form and solved by straightforward elimination for each $n$.

It is convenient to assume that $F_{n}\left(p_{0}\right)=0$. This involves no loss of generality, since it is possible to absorb the right-hand side of $(2.14 \mathrm{~b})$ into the right-hand side of (2.13) through an appropriate redefinition of the dependent variable $\omega_{n}$. It may then be seen that, for each Hough mode, the relative importance of the surface pressure tendency may be estimated by the ratio of the second term on the left-hand side of $(2.14 \mathrm{~b})$ to the first, namely by the dimensionless parameter

$$
\frac{4 \Omega^{2} a^{2} \rho_{\mathrm{o}} \lambda_{n}}{p_{\mathrm{o}}\left|\epsilon_{n}\right|}=\frac{4 \Omega^{2} a^{2} \lambda_{n}}{R T_{\mathrm{o}}\left|\epsilon_{n}\right|}=\frac{4 \Omega^{2} a^{2} \lambda_{n}}{g H\left|\epsilon_{n}\right|}
$$

where $T_{\mathrm{o}}$ is the surface temperature, $g$ the gravitational acceleration, $H=R T_{\mathrm{o}} / g$ the density scale height, and where the vertical derivative has been estimated according to $d / d p \sim \lambda_{n} / p$.

The expression (2.15) appears to depend both on the horizontal scale $L=a\left|\epsilon_{n}\right|^{-1 / 2}$, and on the vertical scale which is manifested through the parameter $\lambda_{n}$. However, these scales are not independent, but are linked through Eq. (2.13). Using the above estimate for $d / d p$, and requiring the homogeneous part of (2.13) to balance, one obtains

$$
\left(4 \Omega^{2} a^{2} / N^{2} H^{2}\right) \lambda_{n}\left(\lambda_{n}-1\right)+\epsilon_{n}=0
$$

relating $\lambda_{n}$ to $\epsilon_{n}$. The static stability parameter $\Gamma$ has been written in terms of the buoyancy frequency $N$ as $\Gamma=N^{2} T_{0} H / g p$. Implicit in the estimate (2.16) is the assumption that $T$, and therefore $N^{2}$, vary only weakly with pressure. Consideration of the solutions of (2.16) and substitution into (2.15) show that the changes in surface pressure must be properly accounted for unless

$$
\frac{4 \Omega^{2} a^{2}}{g H\left|\epsilon_{n}\right|} \max \left\{1, \frac{\left|\epsilon_{n}\right|^{1 / 2} N H}{2 \Omega a}\right\} \ll 1
$$

a condition which does not depend explicitly on $\lambda_{n}$.

Condition (2.17) may be written in the more physically revealing form

$$
\max \left\{M_{n}^{2},\left(N^{2} H / g\right)^{1 / 2} M_{n}\right\} \ll 1
$$

where $M_{n}$ is the rotational Mach number defined by

$$
M_{n}^{2}=4 \Omega^{2} a^{2} / g H\left|\epsilon_{n}\right|=f^{2} L^{2} / g H
$$

taking $f=2 \Omega$. This is equivalent to the condition under which the phase speed of the external Rossby mode is well approximated when the $\omega=0$ boundary condition is applied. It is implicit in the expression (10) of White (1978), for example, where using his notation the approximation would be a good one provided

$$
g H / f^{2} L^{2} \equiv q^{2} \gg \max \{1, Y\} \equiv \max \left\{1, N^{2} H / g\right\} .
$$


Under conventional 'type 1' quasi-geostrophic scaling (e.g. Pedlosky 1964), both $M_{n}$ and $N^{2} H / g$ are taken to be small, in which case (2.18) is satisfied. It is clear that (2.18) will be satisfied for sufficiently small horizontal scale $L$, equivalently for sufficiently large $n$, and it therefore may be justifiable to neglect the effect of the surface pressure tendency on synoptic-scale baroclinic eddies, for example (White and Gadian 1979). However, $M_{n}$ is not particularly small for planetary-scale motions, including those induced by the eddies; substituting typical atmospheric values into (2.19), taking $H=8 \mathrm{~km}$, yields $M_{n} \approx 0.9$ for the second Hough mode $n=2$, so that (2.18) is certainly violated.

Note that, by itself, $M_{n} \ll 1$ is not enough to guarantee that (2.18) hold. Under tropospheric conditions, $N^{2} H / g \approx 0.08$; for an isothermal atmosphere consisting of a diatomic gas, $N^{2} H / g=\kappa=R / c_{p} \approx 2 / 7$. For these cases, then, $M_{n} \ll 1$ is sufficient for (2.18) to hold. However, one could conceive of an atmosphere in which the temperature increased sufficiently rapidly with height that $N^{2} H / g$ was large, and then (2.18) would require that $M_{n} \ll\left(N^{2} H / g\right)^{-1 / 2}$.

The condition (2.17), (2.18) may be written equivalently in terms of a vertical length scale $D$ for non-density-weighted velocities. Estimating $d / d z \sim 1 / D$ and therefore $d / d p \sim H / p D=\lambda_{n} / p$, substitution of $\lambda_{n}=H / D$ into (2.16) yields

$$
L=a /\left|\epsilon_{n}\right|^{1 / 2} \approx \min \left\{N D / 2 \Omega, N(D H)^{1 / 2} / 2 \Omega\right\}
$$

or equivalently

$$
D \sim \max \left\{f L / N, f^{2} L^{2} / N^{2} H\right\} .
$$

The further substitution of (2.21) into (2.18) to eliminate $L$ then gives the requirement

$$
N^{2} D / g \ll 1
$$

under which changes in surface pressure may be safely ignored. This is equivalent to the condition discussed by White (1977), and may also be derived directly by estimating the size of the first term in (2.7) from the hydrostatic and thermodynamic equations alone.

Condition (2.22) is equivalent to a condition that the vertical scale $D$ be much less than the potential-temperature scale height $g / N^{2}$. For a weakly stratified fluid like the atmosphere, where $g / N^{2}$ is significantly greater than the density scale height $H$ (e.g. $g / N^{2} \approx 100 \mathrm{~km}$ using typical tropospheric values), condition (2.22) would appear to be easily satisfied. It must be remembered, however, firstly that $D$ may exceed $H$, and secondly that when $D$ does exceed $H$ it increases quadratically with $L$. Indeed, for the planetary-scale Hough mode $n=2$ considered earlier, $D \sim 80 \mathrm{~km}$.

\section{GENERAL PROPERTIES OF THE INVERSION PROBLEM}

\section{(a) An idealized solution}

In order to gain some insight into the effect on the inversion problem of accounting correctly for the surface pressure tendency, it is instructive to consider an idealized case. in particular, let the atmosphere be isothermal with temperature $T_{0}$, and suppose that the thermal forcing vanishes, while the mechanical forcing takes the form

$$
F(p, \mu)=\alpha \mu B_{2}(\mu) z \exp (-\gamma z)=-\alpha H \mu B_{2}(\mu) \ln \left(p / p_{\mathrm{o}}\right)\left(p / p_{\mathrm{o}}\right)^{\gamma}
$$

as shown in Fig. 1, where $z=-H \ln \left(p / p_{\mathrm{o}}\right)$, and $\alpha$ and $\gamma$ are measures of the amplitude and vertical decay rate of the forcing. The meridional structure of the forcing thus consists of a single mode $n=2$, which means that the response will also be in that mode. The vertical structure equation (2.13) in the isothermal case may be easily solved analytically; 


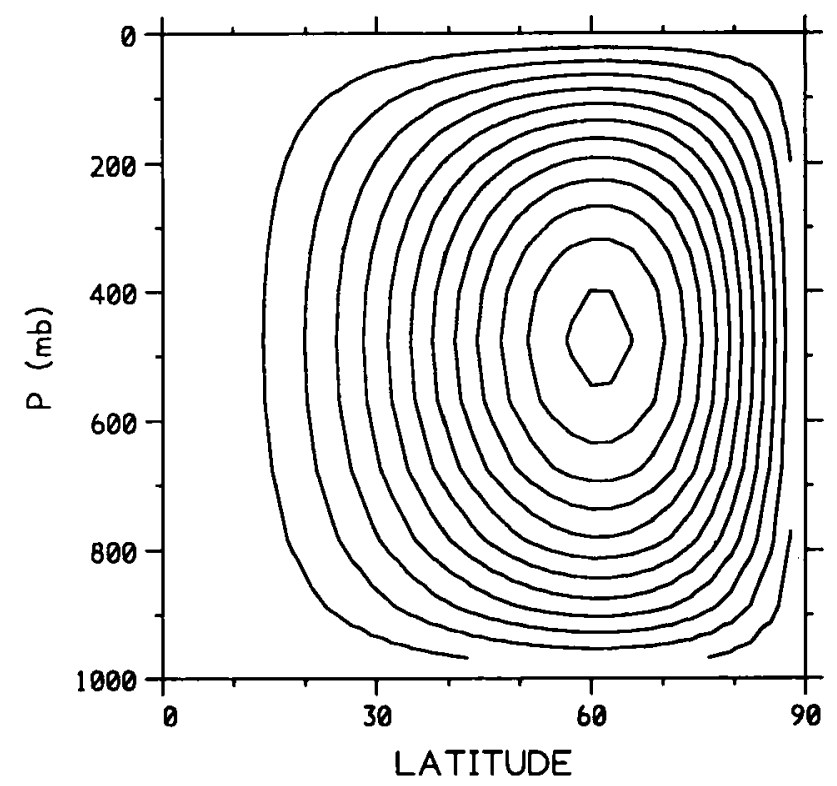

Figure 1. The forcing function (3.1) for the case of peak forcing at $500 \mathrm{mb}$. Peak forcing is $1.7 \times 10^{-6} \mathrm{~m} \mathrm{~s}^{-2}$.

the coefficient $\omega_{2}(p)$ is simply the combination of the homogeneous solution to (2.13) which satisfies (2.14a), namely

$$
\omega_{(h)}=\left(p / p_{0}\right)^{\lambda_{2}^{+}}
$$

and the particular solution of (2.13), namely

$$
\omega_{(p)}=-\frac{p_{0} \alpha H\left(p / p_{0}\right)^{\gamma+1}}{2 \Omega a\left(\lambda_{2}^{+}+\gamma\right)\left(\lambda_{2}^{-}+\gamma\right)}\left\{\gamma \ln \left(p / p_{\mathrm{o}}\right)+\frac{\lambda_{2}^{+} \lambda_{2}^{-}-\gamma^{2}}{\left(\lambda_{2}^{+}+\gamma\right)\left(\lambda_{2}^{-}+\gamma\right)}\right\}
$$

which together will satisfy the lower boundary condition (2.14b). In the above $\lambda_{2}^{+}$and $\lambda_{2}^{-}$are respectively the positive and negative roots of the quadratic equation (2.16) with $n=2$, noting that $N^{2}$ is constant for an isothermal atmosphere.

This idealized case has been studied by running the spherical primitive-equation numerical model of Hoskins and Simmons (1975) with the forcing (3.1), from an initial isothermal state at rest; $\alpha$ was taken to be a smooth function of time increasing from zero (to maintain balance), specifically $\alpha \propto 1-\cos [\pi$ (days)/40]. The resulting evolution can be compared with the numerical solution of the inversion problem defined by (2.13) and (2.14) (which itself was checked against the analytical solution described above), and with the solution obtained by enforcing $\omega=0$ at $p=p_{0}$ (i.e. ignoring the surface pressure tendency). The results for the forcing of Fig. 1 are presented in Fig. 2, which shows the zonal wind acceleration $\partial u / \partial t$ and the streamfunction $\psi$ of the induced meridional circulation, the latter being defined by

$$
v=\left(1-\mu^{2}\right)^{-1 / 2} \partial \psi / \partial p, \quad \omega=-a^{-1} \partial \psi / \partial \mu
$$

The most noticeable feature of the results is the fact that, in the model response, $\omega$ is substantially non-zero at $p=p_{0}$; this confirms that the surface pressure tendency is a leading-order part of the response. Indeed, none of the plotted streamlines are closed, which suggests that the conventional inversion (for which $p=p_{\mathrm{o}}$ is a streamline and all streamlines are closed) is even qualitatively very wrong. The solution of the full inversion 

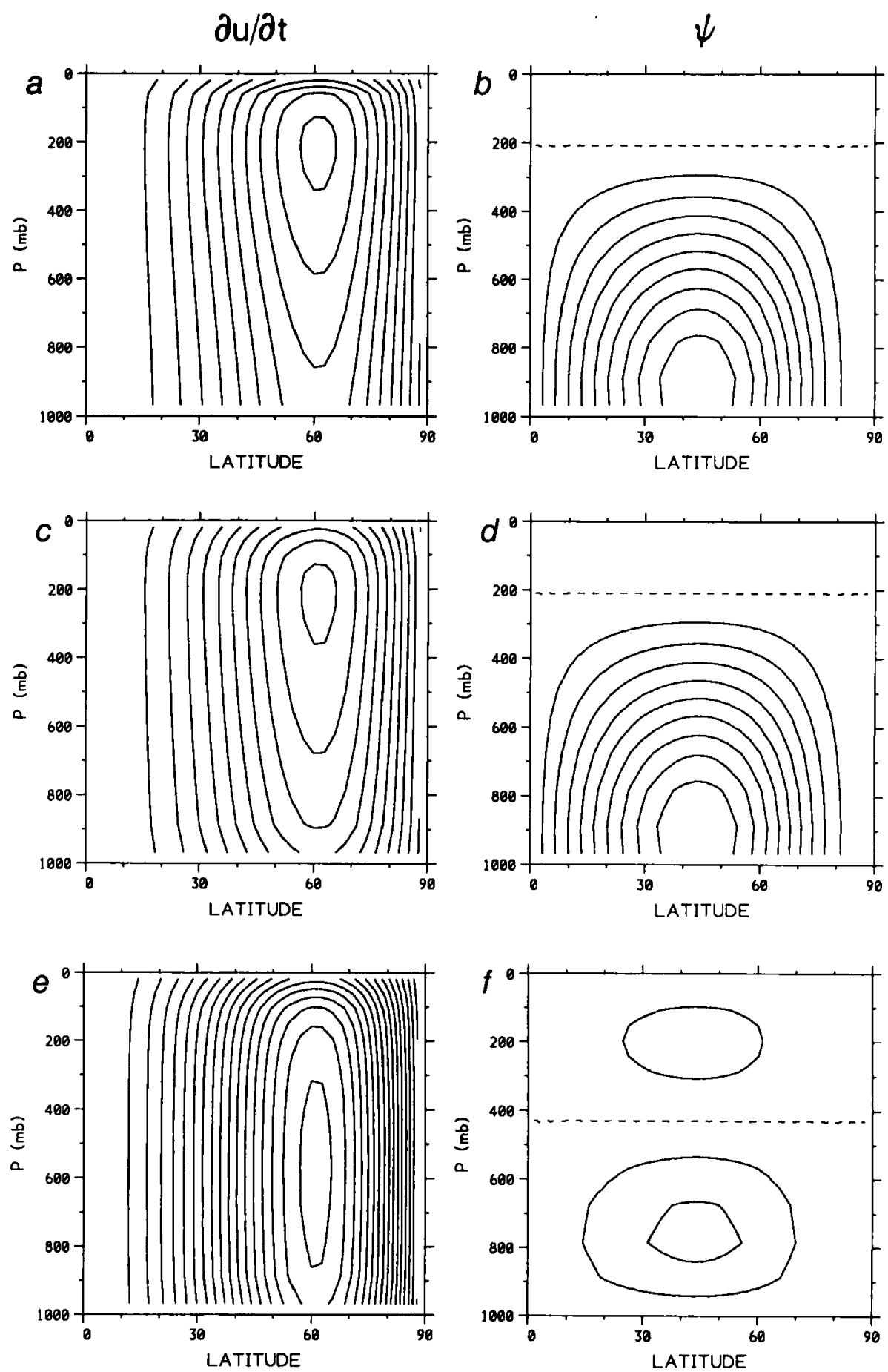

Figure 2. Response to forcing (3.1) of the zonal wind acceleration $\partial u / \partial t$ and the meridional streamfunction $\psi$. (a), (b): Response from a numerical integration of the primitive equations. (c), (d): Predicted response from inversion problem with correct lower boundary condition. (e), (f): Predicted response from inversion problem with surface pressure tendency ignored. The meridional circulation is counter-clockwise in (b) and (d), and in the lower cell in (f). The contour interval for $\partial u / \partial t$ is $2 \times 10^{-8} \mathrm{~m} \mathrm{~s}^{-2}$; that for $\psi$ is $2.5 \times 10^{-4} \mathrm{~m} \mathrm{~s}^{-1} \times 1000 \mathrm{mb}$. 
problem captures the response quite accurately, as it should do since, although the numerical model integrates the full primitive equations in sigma coordinates, the initial conditions and weak amplitude of the forcing were chosen to make the flow quasigeostrophic. The close agreement between these two solutions is therefore a good check that the linearized inversion problem is indeed self-consistently posed. The effect of the non-vanishing surface pressure tendency is also to alter significantly the vertical structure of the zonal wind tendency (the meridional structure being determined here by the modal nature of the forcing). The response subject to $\omega=0$ at $p=p_{\mathrm{o}}$ is seen to be: (i) too strong; (ii) too barotropic (i.e. with insufficient vertical shear); and (iii) with its maximum too low.

Various other experiments were performed with the decay parameter $\gamma$ adjusted to give the peak forcing at different levels. These experiments all confirmed that setting $\omega=0$ at $p=p_{\mathrm{o}}$ in the inversion gives a response that is too strong and too barotropic, as in Fig. 2. The results are summarized in Table 1, which gives the relative magnitude of the peak response with and without the surface pressure tendency.

TABLE 1. RATIO OF PEAK ZONAL, WIND ACCELERATIONS IN THE MODEL RESPONSE TO THE FORCING (3.1)

\begin{tabular}{lcccc}
\hline & \multicolumn{3}{c}{ Pressure level of peak forcing } \\
\cline { 2 - 5 } & $200 \mathrm{mb}$ & $300 \mathrm{mb}$ & $500 \mathrm{mb}$ & $675 \mathrm{mb}$ \\
\hline $\begin{array}{l}\text { Ratio of peak zonal wind acceleration with the } \\
\text { surface pressure effect to that without it }\end{array}$ & 0.79 & 0.71 & 0.59 & 0.53 \\
\hline
\end{tabular}

(b) The Green's function

To unravel the various elements of the response, and in particular the specific effect of a non-vanishing surface pressure tendency, it is instructive to examine the Green's function associated with mechanical forcing. Because of the linear nature of the problem, one may consider the response associated with each meridional mode $n$ separately; then the Green's function for each mode is the solution of the inversion problem (2.13), (2.14) with $F_{n}(p)$ given by

$$
F_{n}(p)=\delta\left(p-p_{1}\right)
$$

with $\delta()$ the Dirac delta function.

One of the striking features seen in the idealized solution presented in Fig. 2 concerned the effect of the surface pressure tendency on the vertical structure of the zonal wind acceleration. In order to focus on this effect most clearly, it is convenient to solve the Green's-function problem for $\partial u / \partial t$ directly instead of for $\omega$. It may be verified that, after eliminating the other dependent variables from (2.1)-(2.5) in favour of $\partial u / \partial t$, one obtains

$$
\begin{aligned}
& \frac{\left(1-\mu^{2}\right)^{1 / 2}}{\mu} \frac{\partial^{2}}{\partial \mu^{2}}\left\{\frac{\left(1-\mu^{2}\right)^{1 / 2}}{\mu} \frac{\partial u}{\partial t}\right\}+4 \Omega^{2} a^{2} \frac{\partial}{\partial p}\left\{\frac{p}{\Gamma R} \frac{\partial}{\partial p} \frac{\partial u}{\partial t}\right\} \\
& =\frac{\left(1-\mu^{2}\right)^{1 / 2}}{\mu} \frac{\partial^{2}}{\partial \mu^{2}}\left\{\frac{\left(1-\mu^{2}\right)^{1 / 2}}{\mu} F\right\}+2 \Omega a \frac{\left(1-\mu^{2}\right)^{1 / 2}}{\mu} \frac{\partial}{\partial p}\left\{\Gamma^{-1} \frac{\partial Q}{\partial \mu}\right\}
\end{aligned}
$$

which is analogous to (2.8). In terms of the coefficients of the expansions (2.11), (3.5) may be written 


$$
\frac{4 \Omega^{2} a^{2}}{R} \frac{d}{d p}\left\{\frac{p}{\Gamma} \frac{d u_{n}}{d p}\right\}+\epsilon_{n} u_{n}=\epsilon_{n} F_{n}+2 \Omega a \epsilon_{n} \frac{d}{d p}\left\{\Gamma^{-1} Q_{n}\right\}
$$

which is a set of ordinary differential equations for $u_{n}(p)$, the Hough-function coefficients for $\partial u / \partial t$. The appropriate constraints on the solutions of (3.6)-no longer strictly boundary conditions-are

$$
\begin{gathered}
p u_{n} \rightarrow 0 \quad \text { as } \quad p \rightarrow 0 \\
\int_{0}^{p_{0}}\left(u_{n}-F_{n}\right) d p=\left(4 \Omega^{2} a^{2} \rho_{\mathrm{o}} / \epsilon_{n}\right) u_{n}\left(p_{\mathrm{o}}\right) .
\end{gathered}
$$

The set (3.6), (3.7) for $u_{n}(p)$ is entirely analogous to (2.13), (2.14) for $\omega_{n}(p)$, and indeed one may derive one from the other by using the original equations (2.1)-(2.5) together with the Galerkin representations (2.11). Equation (3.7b) expresses the angular momentum balance of the system. Its physical interpretation will be discussed later, but it should be evident from (2.15) that the right-hand side is negligible in the limit (2.17), (2.18).

The problem at hand now concerns solving (3.6), (3.7) in an isothermal atmosphere with $Q_{n}=0$ and $F_{n}$ as defined by (3.4). As is customary, one writes $u_{n}$ in terms of the homogeneous solutions of (3.6) both above and below $p=p_{1}$, viz.

$$
\begin{array}{llll}
u_{n}(p)=u_{n<}^{-}\left(p / p_{1}\right)^{-\lambda_{n}^{-}}+u_{n<}^{+}\left(p / p_{1}\right)^{-\lambda_{n}^{+}} & \text {for } & p<p_{1} \\
u_{n}(p)=u_{n>}^{-}\left(p / p_{1}\right)^{-\lambda_{n}^{-}}+u_{n>}^{+}\left(p / p_{1}\right)^{-\lambda_{n}^{+}} & \text {for } & p>p_{1}
\end{array}
$$

where $\lambda_{n}^{+}$and $\lambda_{n}^{-}$are respectively the positive and negative roots of the quadratic (2.16). Now, (3.7a) implies that $u_{n<}^{+}=0$. The other three coefficients are determined by solving the three equations (3.6) (after integrating across $\left.p_{1}\right),(3.7 \mathrm{~b})$, and continuity of the zonal wind tendency

$$
u_{n<}^{-}=u_{n}\left(p_{1}\right)=u_{n>}^{-}+u_{n>}^{+}
$$

For example, it is readily found from (3.6) and (3.9) that

$$
u_{n>}^{+}=\frac{-\epsilon_{n} N^{2} H^{2}}{4 \Omega^{2} a^{2} p_{1}\left(\lambda_{n}^{+}-\lambda_{n}^{-}\right)}=\frac{-\epsilon_{n} N^{2} H^{2}}{4 \Omega a p_{1}\left(\Omega^{2} a^{2}-\epsilon_{n} N^{2} H^{2}\right)^{1 / 2}}
$$

which is positive since the eigenvalues $\epsilon_{n}$ are always negative. Note that $(3.7 \mathrm{~b})$ is not needed to obtain (3.10). Considering the case where the forcing is far removed from the lower boundary, namely the limit $p_{1} \rightarrow 0$, one expects $u_{n>}^{-}=0$ (i.e. no upwards-decaying part to $u_{n}$ below $p=p_{1}$ ) and $u_{n<}^{-}=u_{n>}^{+}$; thus $u_{n}(p)$ simply falls off algebraically from $p=p_{1}$, though more quickly for $p>p_{1}$ than for $p<p_{1}$ since the modulus of $\lambda_{n}^{+}$always exceeds that of $\lambda_{n}^{-}$. For a general vertical distribution of $F_{n}(p)$, when a number of such solutions, with different values of $p_{1}$, are superposed, there will be a tendency for the response to peak above the level of peak forcing.

In order to consider the effect of the lower boundary, it is convenient to divide the response into three different parts: (1) that which would be obtained in the limit $p_{1} \rightarrow 0$, where the choice of the lower boundary condition is immaterial; (2) that due to the finite value of $p_{1}$ (i.e. allowing an upwards-decaying part to $u_{n}$ below $\left.p=p_{1}\right)$, but with $\omega\left(p_{o}\right)=$ 0 instead of the proper boundary condition; and (3) that due to the surface pressure tendency. These solution components shall be denoted by the associated numbers as arguments, for example

$$
u_{n>}^{+}=u_{n>}^{+}(1)+u_{n>}^{+}(2)+u_{n>}^{+}(3), \quad u_{n}(p)=u_{n}(1 ; p)+u_{n}(2 ; p)+u_{n}(3 ; p)
$$


As already discussed,

$$
u_{n>}^{+}(1)=\frac{-\epsilon_{n} N^{2} H^{2}}{4 \Omega^{2} a^{2} p_{1}\left(\lambda_{n}^{+}-\lambda_{n}^{-}\right)}>0, \quad u_{n>}^{+}(2)=0, \quad u_{n>}^{+}(3)=0 .
$$

Solving the system of three equations as described above, and taking the relevant limits to obtain the different components (1)-(3), the other components are found to be

$$
\begin{aligned}
& u_{n>}^{-}(1)=0, \quad u_{n>}^{-}(2)=-\frac{\lambda_{n}^{+}}{\lambda_{n}^{-}}\left(p_{1} / p_{0}\right)^{\left(\lambda_{n}^{+}-\lambda_{n}^{-}\right)} u_{n>}^{+}(1)>0 \\
& u_{n>}^{-}(3)=-\frac{N^{2} H^{2}\left(\lambda_{n}^{+}-\lambda_{n}^{-}\right)}{\lambda_{n}^{-}\left(R T_{0} \lambda_{n}^{-}-N^{2} H^{2}\right)}\left(p_{1} / p_{0}\right)^{\left(\lambda_{n}^{+}-\lambda_{n}^{-}\right)} u_{n>}^{+}(1)<0 \\
& u_{n<}^{-}(1)=u_{n>}^{+}(1), \quad u_{n<}^{-}(2)=u_{n>}^{-}(2), \quad u_{n<}^{-}(3)=u_{n>}^{-}(3) .
\end{aligned}
$$

(Note that it is the fact that the denominator of (3.11c) cannot vanish that rules out the possibility of a non-trivial free solution arising as a result of using the boundary condition (2.9) rather than $\omega\left(p_{0}\right)=0$.) Thus the correction due to finite $p_{1}$, denoted (2), is always such as to add a positive upwards-decaying part to $u_{n}(p)$, and thereby to make the response both stronger and more barotropic; whereas the correction due to the proper lower boundary condition, denoted (3), is to add a negative upwards-decaying part, and thus to weaken the response and make it more baroclinic. This accounts, at least qualitatively, for the features remarked on concerning Fig. 2. The various effects of these contributions are indicated schematically in Fig. 3.
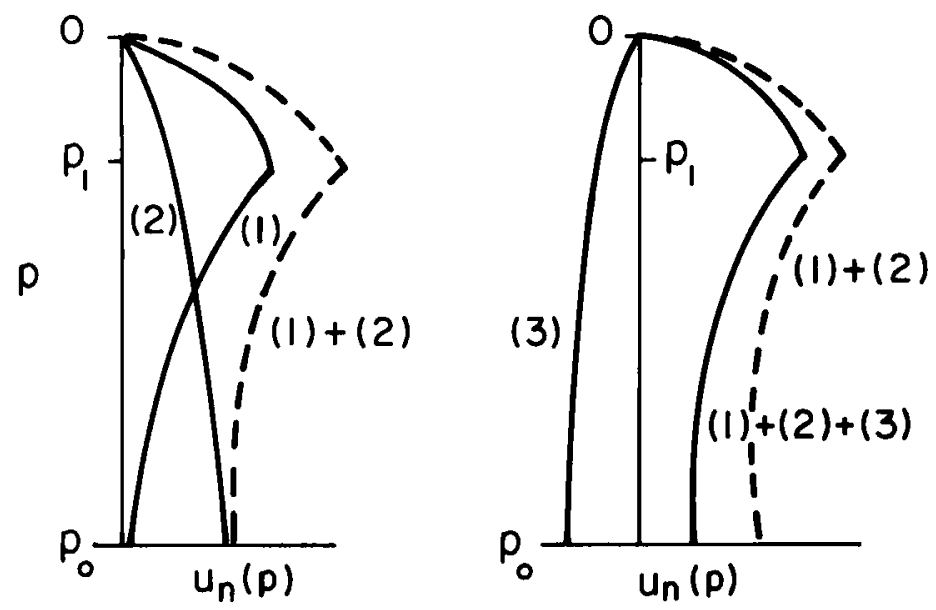

Figure 3. Schematic of the vertical profiles of the Hough-function coefficients $u_{n}(p)$ of the zonal wind acceleration, corresponding to the different solution components: (1) no lower-boundary effects; (2) finite $p_{1}$ but no surface pressure effect; and (3) surface pressure effect. It is evident that $(1)+(2)$ is too strong and too barotropic when compared with the correct profile $(1)+(2)+(3)$.

\section{(c) Characterization of the effects due to surface pressure changes}

When the condition $\omega\left(p_{0}\right)=0$ is imposed in the response problem, it follows that the mean meridional circulation forms a closed cell and the net meridional mass flux across any latitude circle must vanish. This means that, at each latitude, the net relative angular momentum tendency must equal the net forcing, as is evident from the vertical 
integral of (2.1). The sum of contributions (1) and (2) above is subject to this constraint, and one may indeed verify from (3.8) and (3.11) that

$$
\int_{0}^{p_{\mathrm{o}}}\left\{u_{n}(1 ; p)+u_{n}(2 ; p)\right\} d p=\int_{0}^{p_{\mathrm{o}}} F_{n}(p) d p=1
$$

By allowing $\omega\left(p_{\mathrm{o}}\right)$ to be non-zero, however, a net meridional mass flux becomes possible and the net relative angular momentum tendency can differ from the net forcing. By (3.12), this difference is entirely contained in the vertical integral of $u_{n}(3 ; p)$; by using (3.8) and (3.11) it is found to be

$$
\int_{0}^{p_{0}} u_{n}(3 ; p) d p=\frac{p_{1} u_{n>}^{-}(3)}{\left(1-\lambda_{n}^{-}\right)}\left(p_{\mathrm{o}} / p_{1}\right)^{\lambda_{n}^{+}}=-\frac{N^{2} H^{2}}{N^{2} H^{2}-R T_{\mathrm{o}} \lambda_{n}^{-}}\left(p_{1} / p_{\mathrm{o}}\right)^{-\lambda_{n}^{-}}
$$

which is negative and has a modulus less than unity. Table 2 gives the ratio of the vertically-integrated mean-flow acceleration with the surface pressure effect, (3.12) plus (3.13), to that without it, (3.12) (which is unity).

TABLE 2. SOME MEASURES OF THE MAGNITUDE AND VERTICAL STRUCTURE OF THE ZONAL WIND ACCELERATION

\begin{tabular}{|c|c|c|c|c|c|}
\hline & \multicolumn{5}{|c|}{ Pressure level of forcing } \\
\hline & $200 \mathrm{mb}$ & $300 \mathrm{mb}$ & $500 \mathrm{mb}$ & $675 \mathrm{mb}$ & $800 \mathrm{mb}$ \\
\hline $\begin{array}{l}\text { Ratio of vertically-integrated accelerations } \\
\text { Eqs. }(3.12)+(3.13)\end{array}$ & $0 \cdot 61$ & 0.58 & 0.53 & $0 \cdot 49$ & 0.47 \\
\hline $\begin{array}{l}\text { Ratio of peak accelerations } \\
\text { Eq. }(3.20)\end{array}$ & 0.78 & 0.69 & 0.57 & $0 \cdot 51$ & 0.48 \\
\hline $\begin{array}{l}\text { Vertical variation without the } \\
\text { surface pressure effect Eq. (3.21) }\end{array}$ & 1.73 & 1.33 & 1.09 & 1.03 & 1.01 \\
\hline $\begin{array}{l}\text { Vertical variation with the surface } \\
\text { pressure effect Eq. (3.22) }\end{array}$ & 3.01 & $2 \cdot 05$ & $1 \cdot 38$ & $1 \cdot 16$ & 1.08 \\
\hline
\end{tabular}
IN RESPONSE TO THE FORCING (3.4), MODE 2

The above demonstrates that

$$
0<\int_{0}^{p_{0}} u_{n}(p) d p<\int_{0}^{p_{0}} F_{n}(p) d p
$$

a result which generalizes (by linear superposition) to arbitrary positive forcing profiles $F_{n}(p)$, with the obvious counterpart for negative forcing. The physical content of (3.14) is that only part of the mechanical forcing goes into mean-flow acceleration, the rest generating an increase in planetary angular momentum through a net equatorward meridional mass flux. Similar comments apply to the condition (3.7b) discussed earlier.

The negative root $\lambda_{n}^{-}$of (2.16) may be written as

$$
\lambda_{n}^{-}=\frac{1}{2}\left[1-\left\{1-\frac{\epsilon_{n} N^{2} H^{2}}{\Omega^{2} a^{2}}\right\}^{1 / 2}\right]=\frac{1}{2}\left[1-\left\{1+\frac{4 K}{M_{n}^{2}}\right\}^{1 / 2}\right]
$$

with $M_{n}$ given by (2.19), where the fact that $N^{2} H / g=\kappa \equiv R / c_{p}$ for an isothermal atmosphere has been used. It follows from (3.15) that

$$
-\lambda_{n}^{-} \sim \min \left\{\frac{\kappa}{M_{n}^{2}}, \frac{\kappa^{1 / 2}}{M_{n}}\right\}
$$


In the quasi-Boussinesq limit (2.18), one has

$$
\max \left\{M_{n}^{2}, \kappa^{1 / 2} M_{n}\right\} \ll 1
$$

and so combining (3.16) and (3.17) yields

$$
\frac{\kappa}{-\lambda_{n}^{-}} \sim\left[\min \left\{M_{n}^{-2}, \kappa^{-1 / 2} M_{n}^{-1}\right\}\right]^{-1}=\max \left\{M_{n}^{2}, \kappa^{1 / 2} M_{n}\right\} \ll 1
$$

whence

$$
\frac{N^{2} H^{2}}{N^{2} H^{2}-R T_{\mathrm{o}} \lambda_{n}^{-}}=\frac{1}{1-\kappa^{-1} \lambda_{n}^{-}} \sim \min \left\{1, \frac{\kappa}{-\lambda_{n}^{-}}\right\} \ll 1
$$

using $H=R T_{\mathrm{o}} / g$. So in this limit the change due to the surface pressure tendency, (3.13), is negligible when compared with (3.12), as expected. On the other hand, when (2.18) is not satisfied, then $-K^{-1} \lambda_{n}^{-}=O(1)$ and whether the ratio of (3.13) to (3.12) is small or not, is determined by the height of the forcing, $\left(p_{1} / p_{0}\right)^{-\lambda_{n}^{-}}$. Taking $\kappa=2 / 7$ and $M_{n} \approx 0.9$ for typical atmospheric values and the $n=2$ mode, as before, one finds $\lambda_{n}^{-} \approx-0.23$ and $\left(1-\kappa^{-1} \lambda_{n}^{-}\right)^{-1} \approx 0.55$, suggesting a very strong effect, with only a very slow reduction as $p_{1} \rightarrow 0$. For a forcing centred at $200 \mathrm{mb},(3.13)$ is then approximately one third. This implies that only two thirds of the forcing goes into mean-flow acceleration, the other third being expended in driving a net meridional mass flux.

As is evident from Fig. 3, the surface pressure effect reduces not only the verticallyintegrated $\partial u / \partial t$, but also its peak value. The ratio of the peak values, with and without the surface pressure effect, is given by the ratio of the relevant $u_{n}$ s at $p=p_{1}$, namely

$$
\frac{\left\{u_{n}(1)+u_{n}(2)+u_{n}(3)\right\}\left(p_{1}\right)}{\left\{u_{n}(1)+u_{n}(2)\right\}\left(p_{1}\right)}=\frac{u_{n>}^{+}(1)+u_{n>}^{-}(2)+u_{n>}^{-}(3)}{u_{n>}^{+}(1)+u_{n>}^{-}(2)} .
$$

Since $u_{n>}^{+}(1)$ and $u_{n>}^{-}(2)$ are positive and $u_{n>}^{-}(3)$ is negative, the ratio (3.20) is always less than unity. Values of this ratio are given in Table 2 for various values of $p_{1}$. The extent to which these are less than unity is a measure of the importance of the surface pressure effect. The ratios of the peak accelerations may be compared with those in Table 1 for the idealized solution of section $3(a)$, and are seen to be very similar.

The solutions found thus far also point to an important effect on the vertical structure, namely that the mean-flow acceleration is too barotropic when the surface pressure tendency is ignored. A measure of the vertical variation is given by the ratio of the peak acceleration to its value at the surface. Without the surface pressure effect, this ratio is given by

$$
\frac{\left\{u_{n}(1)+u_{n}(2)\right\}\left(p_{1}\right)}{\left\{u_{n}(1)+u_{n}(2)\right\}\left(p_{0}\right)}=\frac{u_{n>}^{+}(1)+u_{n>}^{-}(2)}{u_{n}^{+}(1)\left(p_{1} / p_{0}\right)^{\lambda_{n}^{+}}+u_{n>}^{-}(2)\left(p_{1} / p_{0}\right)^{\lambda_{n}^{-}}}
$$

whereas with the surface pressure effect, the ratio is

$$
\frac{\left\{u_{n}(1)+u_{n}(2)+u_{n}(3)\right\}\left(p_{1}\right)}{\left\{u_{n}(1)+u_{n}(2)+u_{n}(3)\right\}\left(p_{0}\right)}=\frac{u_{n>}^{+}(1)+u_{n>}^{-}(2)+u_{n>}^{-}(3)}{u_{n>}^{+}(1)\left(p_{1} / p_{0}\right)^{\lambda_{n}^{+}}+\left\{u_{n>}^{-}(2)+u_{n>}^{-}(3)\right\}\left(p_{1} / p_{\mathrm{o}}\right)^{\lambda_{n}^{-}}} .
$$

It may be checked from (3.11) that, as expected, (3.22) approaches (3.21) in the quasiBoussinesq limit (2.18), and both ratios increase without bound in the limit $p_{1} \rightarrow 0$. The ratios (3.21) and (3.22) are always greater than unity; the closer they are to unity, the more barotropic is the response. Table 2 displays the ratios for various values of $p_{1}$; as anticipated, (3.21) is always closer to unity than is (3.22). 
TABLE 3. SOME MEASURES OF THE MAGNITUDE AND VERTICAL STRUCTURE OF THE ZONAL. WIND ACCELERATION IN RESPONSE TO THE FORCING (3.4), MODE 5

\begin{tabular}{|c|c|c|c|c|c|}
\hline & \multicolumn{5}{|c|}{ Pressure level of forcing } \\
\hline & $200 \mathrm{mb}$ & $300 \mathrm{mb}$ & $500 \mathrm{mb}$ & $675 \mathrm{mb}$ & $800 \mathrm{mb}$ \\
\hline $\begin{array}{l}\text { Ratio of vertically-integrated accelerations } \\
\text { Eqs. (3.12) }+(3.13)\end{array}$ & 0.95 & 0.93 & $0 \cdot 88$ & 0.84 & 0.81 \\
\hline $\begin{array}{l}\text { Ratio of peak accelerations } \\
\text { Eq. }(3.20)\end{array}$ & 0.99 & 0.98 & 0.93 & 0.86 & $0 \cdot 82$ \\
\hline $\begin{array}{l}\text { Vertical variation without the } \\
\text { surface pressure effect Eq. }(3.21)\end{array}$ & $7 \cdot 77$ & $3 \cdot 67$ & $1 \cdot 62$ & $1 \cdot 17$ & $1 \cdot 05$ \\
\hline $\begin{array}{l}\text { Vertical variation with the surface } \\
\text { pressure effect } \mathrm{Eq} \text {. (3.22) }\end{array}$ & $10 \cdot 04$ & $4 \cdot 67$ & 1.95 & $1 \cdot 31$ & $1 \cdot 12$ \\
\hline
\end{tabular}

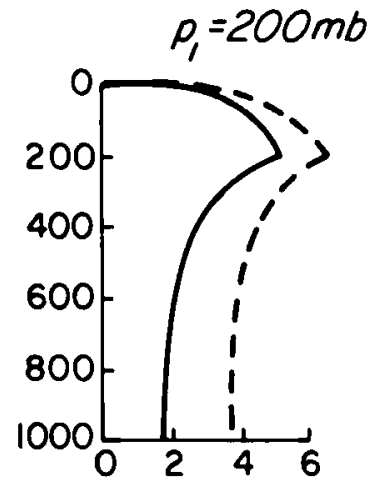

$p_{1}=300 \mathrm{mb}$

$p_{1}=500 \mathrm{mb}$

$\rho_{1}=800 \mathrm{mb}$
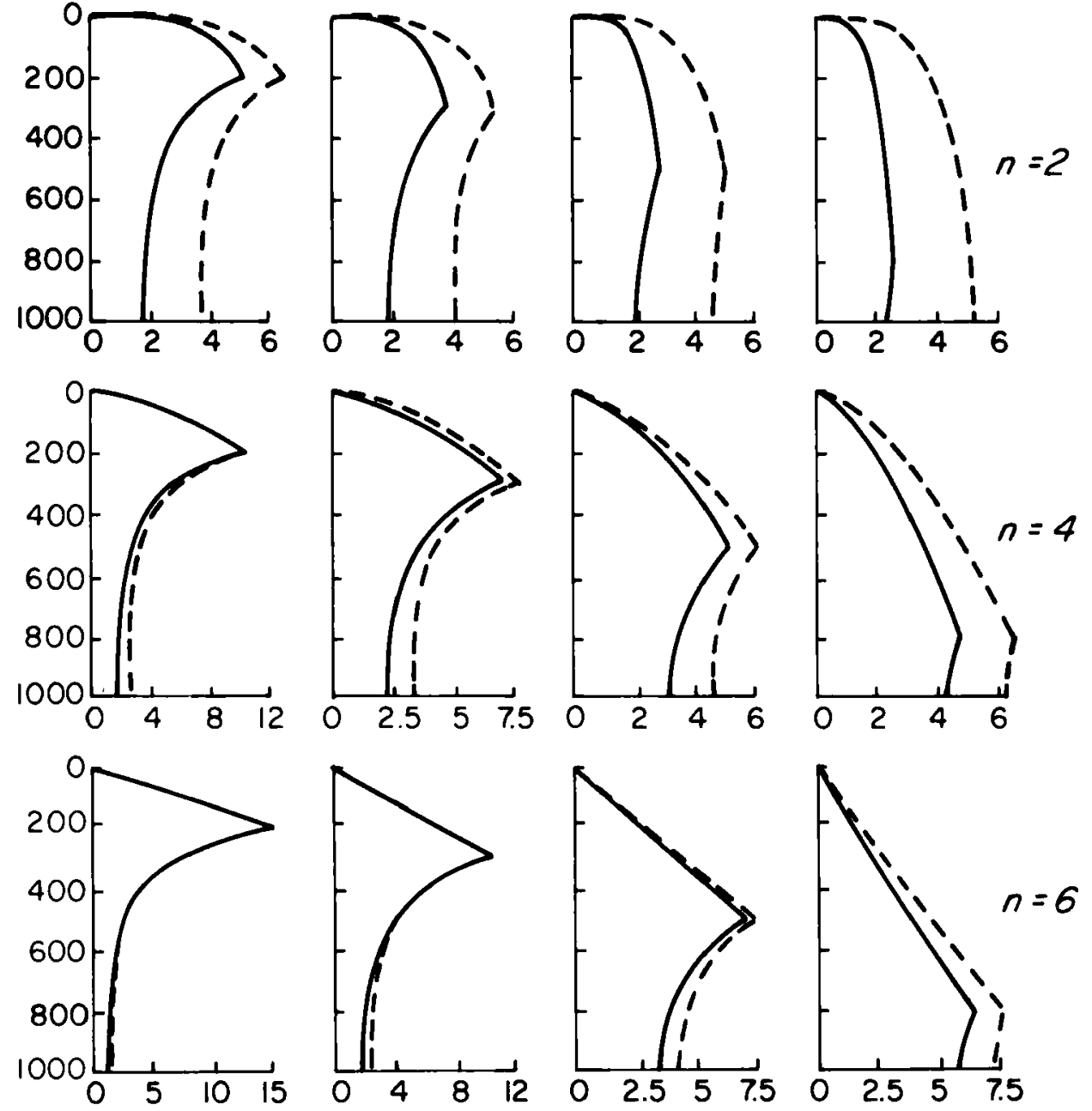

Figure 4. Vertical profiles of the Hough-function coefficients $u_{n}(p)$ of the zonal wind acceleration corresponding to the forcing (3.4), for various forcing levels $p_{1}$ and horizontal mode numbers $n$, as indicated. The solid line is with the surface pressure effect, the dashed line is without. Units are arbitrary but are the same for all cases shown, and so the vertical integral of the dashed line (which equals the net forcing) is a constant. 
On the basis of (2.17), one expects the surface pressure effect to decrease rapidly with increasing mode index $n$. This is confirmed by Table 3 , which shows the same information as Table 2 but for $n=5$ rather than $n=2$.

Vertical profiles of zonal wind acceleration, $u_{n}(p)$, are shown in Fig. 4 for various values of the forcing level $p_{1}$ and the mode index $n$. In each case the profile is shown both with and without the surface pressure effect. The various effects discussed already are exhibited quite clearly. As $n$ increases, the response becomes more strongly confined in the vertical. Hence for moderate $n$ the surface pressure effect is unimportant except for low-level forcing. But for $n=2$, the vertical confinement is so weak that the response is strongly affected by surface pressure changes, even for forcing at upper levels.

It should be noted that the exponent $-\lambda_{n}^{-}$, given by (3.15), which characterizes the vertical confinement of the response, depends on the static stability $N^{2}$. For the isothermal basic state considered in this section, $N^{2}=\mathrm{kg} / H \approx 3.6 \times 10^{-4} \mathrm{~s}^{-2}$. A more realistic tropospheric value of $N^{2}$ is $10^{-4} \mathrm{~s}^{-2}$; using this would reduce $-\lambda_{n}^{-}$and therefore weaken the vertical confinement. Particularly for the higher- $n$ modes, then, Fig. 4 would represent an underestimate of the importance of surface pressure changes associated with tropospheric forcing.

\section{MEAN-FLOW CHANGES IN A BAROCLINIC LIFE-CYCLE}

The previous section has explored the nature of the mean-flow response to idealized forms of mechanical forcing. In this section the inversion algorithm is applied to the important (albeit still somewhat idealized) case of the forcing associated with a baroclinic life-cycle. An understanding of mean-flow changes induced by baroclinic eddies is essential to an understanding of the general circulation itself.

Attention is restricted here to a single case study, using the fully nonlinear, primitiveequation spherical model of Hoskins and Simmons (1975) (with imposed wave-6 and hemispheric symmetry), starting with a zonal-mean state corresponding to the basic case of Simmons and Hoskins (1980), together with a wave-6 initial disturbance, and integrating for 15 days. Details of such baroclinic life-cycles may be found in Simmons and Hoskins $(1978,1980)$; briefly, the disturbance grows exponentially at first, much like a Charney baroclinic mode, until it saturates (about day 6 in this case), after which time it decays barotropically (see also the discussion in Hoskins 1983). The Eliassen-Palm flux divergence is shown in Figs. 5(a), (b) for the two periods 0-6 days and 6-12 days, and is associated with the by-now-familiar upwards and then equatorwards flux of E-P wave activity. The associated mechanical and thermal forcings

$$
\begin{gathered}
F=-a^{-1}\left(1-\mu^{2}\right)^{-1 / 2} \frac{\partial}{\partial \mu}\left\{\left(1-\mu^{2}\right) \overline{u^{\prime} v^{\prime}}\right\} \\
Q=-a^{-1} \frac{\partial}{\partial \mu}\left\{\left(1-\mu^{2}\right)^{1 / 2} \overline{v^{\prime} T^{\prime}}\right\}
\end{gathered}
$$

are also shown in Fig. 5.

Note that $F$ and $Q$ contain all the forcing information, and appear separately as forcings on the right-hand side of the momentum and heat equations (2.1) and (2.4). One may, however, adopt the transformed Eulerian-mean (TEM) approach (Andrews and McIntyre 1976), in which case the two forcing terms are combined in the E-P flux divergence, but it is important to remember that the lower boundary condition on the meridional circulation then depends on the value of $Q$ at the surface. This inhomogeneous 
Days 0-6
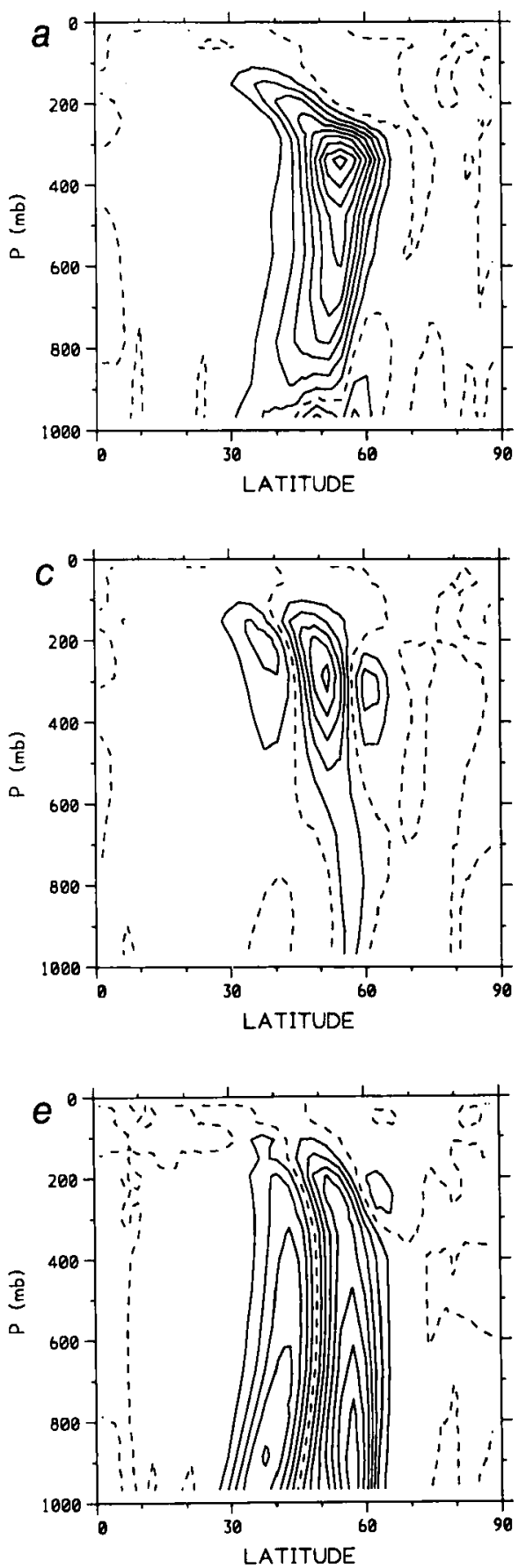

Days 6-12
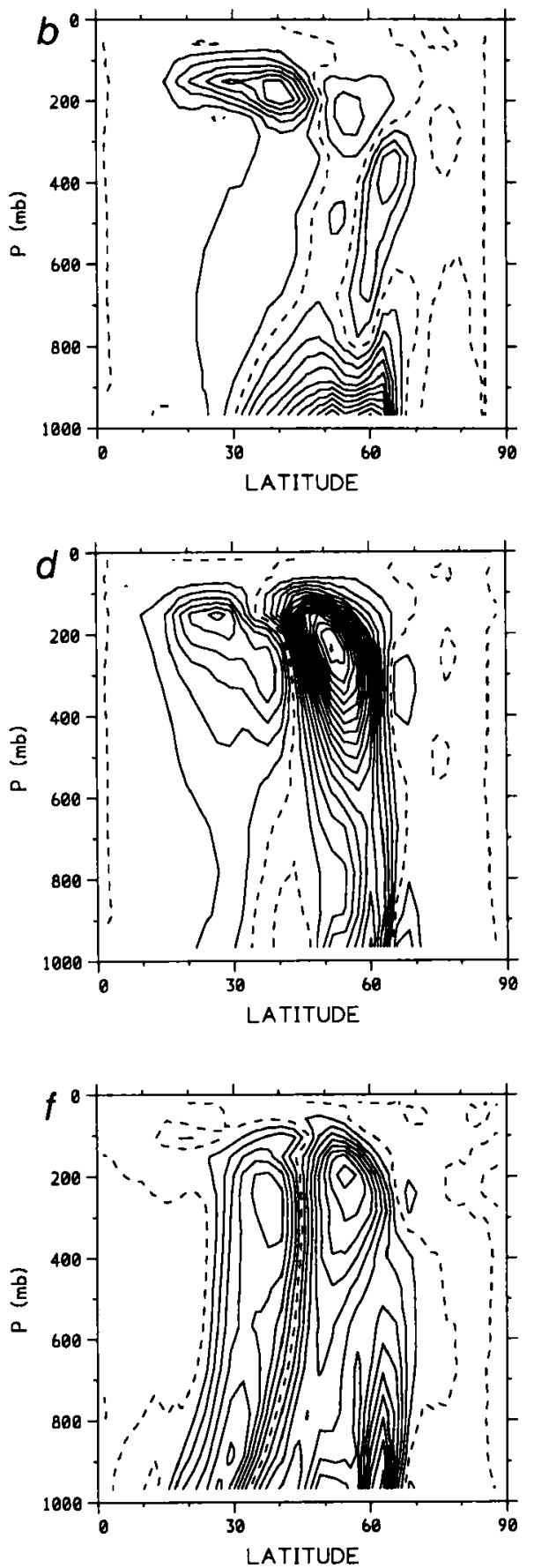

Figure 5. Forcing terms produced by a numerical simulation of a baroclinic life-cycle using a primitiveequation model, for the two periods 0-6 days and 6-12 days. (a), (b): Eliassen-Palm flux divergence, divided by $\cos$ (latitude), which gives an equivalent force per unit mass, contour interval $2 \times 10^{-5} \mathrm{~m} \mathrm{~s}^{-2}$. (c), (d): Mechanical forcing $F$ as defined by (4.1), contour interval $10^{-5} \mathrm{~m} \mathrm{~s}^{-2}$. (e), (f): Thermal forcing $Q$ as defined by (4.2), contour interval $10^{-5} \mathrm{~K} \mathrm{~s}^{-1}$. The zero contours are dashed. The E-P flux divergence is mainly negative except at the lower boundary; $F$ is positive in the principal region around $50^{\circ} \mathrm{N}$; and $Q$ is positive on the poleward side and negative on the equatorward side. 
boundary condition itself represents part of the forcing in the TEM formulation (and thereby renders it slightly less attractive for tropospheric studies).

It may be seen from Fig. 5 that it is perhaps somewhat of an oversimplification to characterize the growth and decay stages of the life-cycle as being baroclinic and barotropic respectively. The thermal and mechanical forcings both seem to be fairly significant during each stage. It is possible that taking a shorter time period for the growth stage might reduce the role of the mechanical forcing, but it appears difficult to remove the contribution of the thermal forcing to the second stage. This is manifested in the E-P flux arrows for day 8 shown in Fig. 7.5(c) of Hoskins (1983), which are far from horizontal.

The meridional circulations and mean-flow tendencies may now be calculated using the algorithm set out in section 2 , subject to imposed hemispheric symmetry as in the model. The inversion is done for each day of the model integration, using the forcings (4.1) and (4.2), and taking the static stability parameter $\Gamma(p)$ to be the globally-averaged $\Gamma$ on each pressure level for that day. Figures 6-8 show the results for the three time periods 0-6 days, 6-12 days and 0-15 days, respectively. Each figure shows the timeaveraged zonal wind acceleration and meridional circulation as produced by the model, and as predicted by the quasi-geostrophic response algorithm both with and without the surface pressure effect.

Looking first at Fig. 6, it is evident from Fig. 6(a) that the effects of $F$ and $Q$ on $\partial u / \partial t$ tend to cancel each other to a certain extent in the upper half of the atmosphere. One may understand this cancellation by noting that, from (3.5), the local maximum in $F$ at $300 \mathrm{mb}$ seen in Fig. 5(c) is opposed, in its effect on $\partial u / \partial t$, by the thermal forcing $Q$, the meridional gradient of which is decreasing with height (Fig. 5(e)). (Note that the same cancellation does not necessarily hold as far as the other response variables are concerned; contrast the right-hand sides of (3.5) and (2.8), for instance.) The main response in $\partial u / \partial t$ is at the surface, and is evidently associated with the strong low-level heat flux; its rapid decay with height is consistent with the nature of the vertical profiles above the forcing levels in Fig. 4. The meridional circulation exhibits the three-cell structure, with an indirect cell in the unstable region, that is characteristic of linear baroclinic instability (e.g. Phillips 1954).

Comparing Figs. 6(c), (e) with 6(a), and 6(d), (f) with 6(b), it may be seen that the quasi-geostrophic inversion captures the gross features of the model response quite well. The main difference in the $\partial u / \partial t$ fields is that the inversions overestimate the strength of the response; the maximum is too large by about $10 \%$ with the correct lower boundary condition (Fig. 6(c)), and by about $30 \%$ with the surface pressure tendency neglected (Fig. 6(e)). Clearly, then, inclusion of the surface pressure effect is crucial if one is to have any hope of getting the correct answer. The major systematic discrepancy in the meridional circulation is that the inversions give a more asymmetric pattern, with the tropical cell significantly stronger than the polar one, whereas in the model the outer cells are more nearly equal. The extent of the net equatorwards mass flux over the unstable region seems to be well captured by the correct inversion (Figs. 6(b), (d)); and is of course completely absent in the other inversion (Fig. 6(f)).

The mean-flow response during days 6-12 is markedly different from that during days $0-6$. The zonal wind acceleration is much less baroclinic, and now extends through the depth of the troposphere (Fig. 7(a)), while the meridional circulation has two cells instead of three (Fig. 7(b)). Examination of the relevant forcing fields for this period (Figs. 5(d), (f)) reveals that there is no particular cancellation at the tropopause level, as there was during days $0-6$, and that the bulk of the forcing is in fact concentrated there. The agreement in the $\partial u / \partial t$ fields between the inversions and the model is rather 

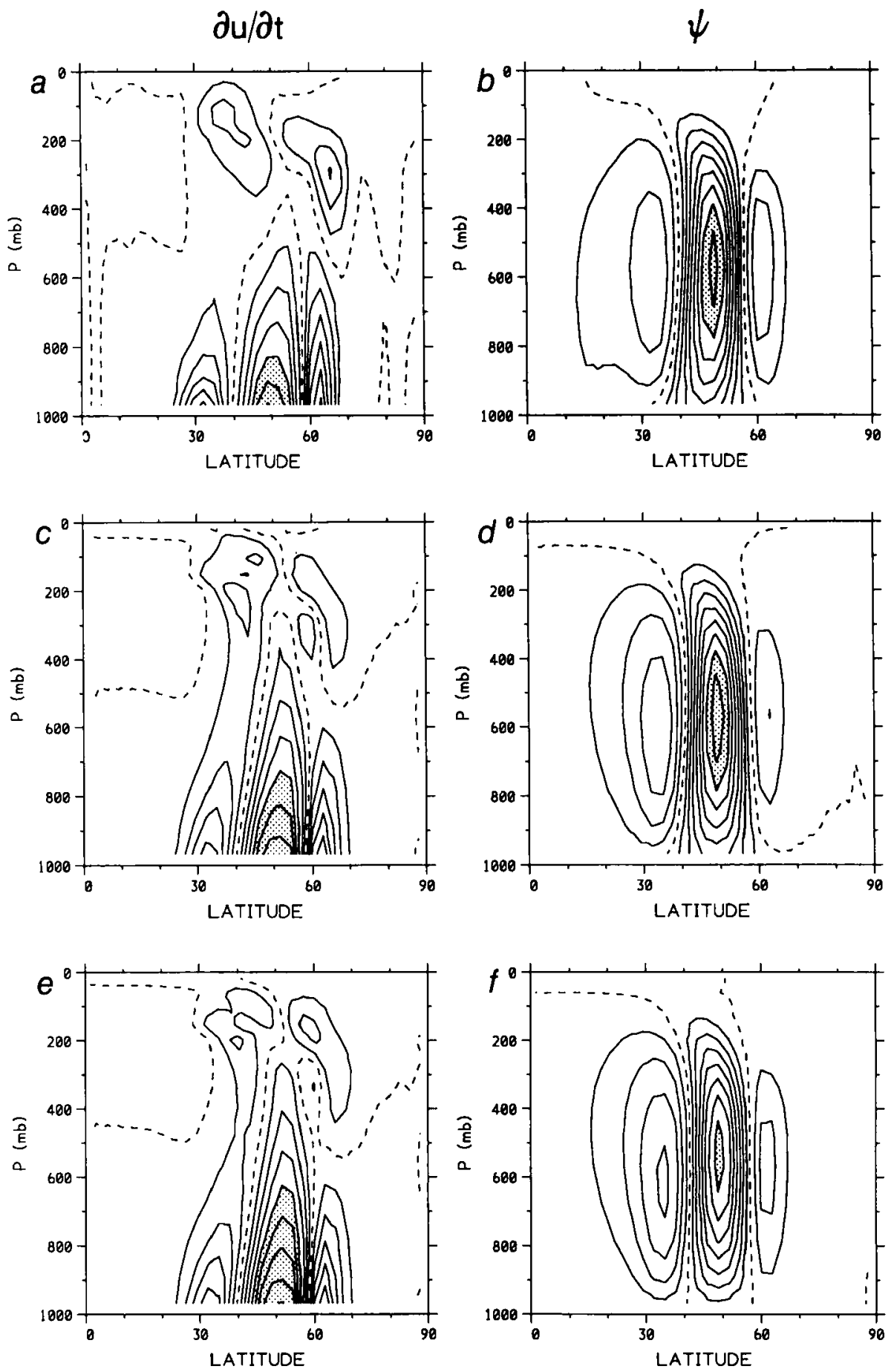

Figure 6. Mean-flow response to a baroclinic life-cycle during the growth stage, days 0-6, showing zonal wind acceleration $\partial u / \partial t$ and meridional streamfunction $\psi$. (a), (b): Model response. (c), (d): Predicted response from inversion problem with correct lower boundary condition. (e), (f): Predicted response from inversion problem with $\omega=0$ at the lower boundary. The contour interval for $\partial u / \partial t$ is $5 \times 10^{-6} \mathrm{~m} \mathrm{~s}^{-2}$; that for $\psi$ is $10^{-2} \mathrm{~m} \mathrm{~s}^{-1} \times 1000 \mathrm{mb}$. Stippled regions represent $\partial u / \partial t$ greater than $2 \times 10^{-5} \mathrm{~m} \mathrm{~s}^{-2}$ and $\psi$ less than $-7 \times 10^{-2} \mathrm{~m} \mathrm{~s}^{-1} \times 1000 \mathrm{mb}$. The central extremum of $\partial u / \partial t$ at the ground is positive, and the central cell for $\psi$ is counter-clockwise. 

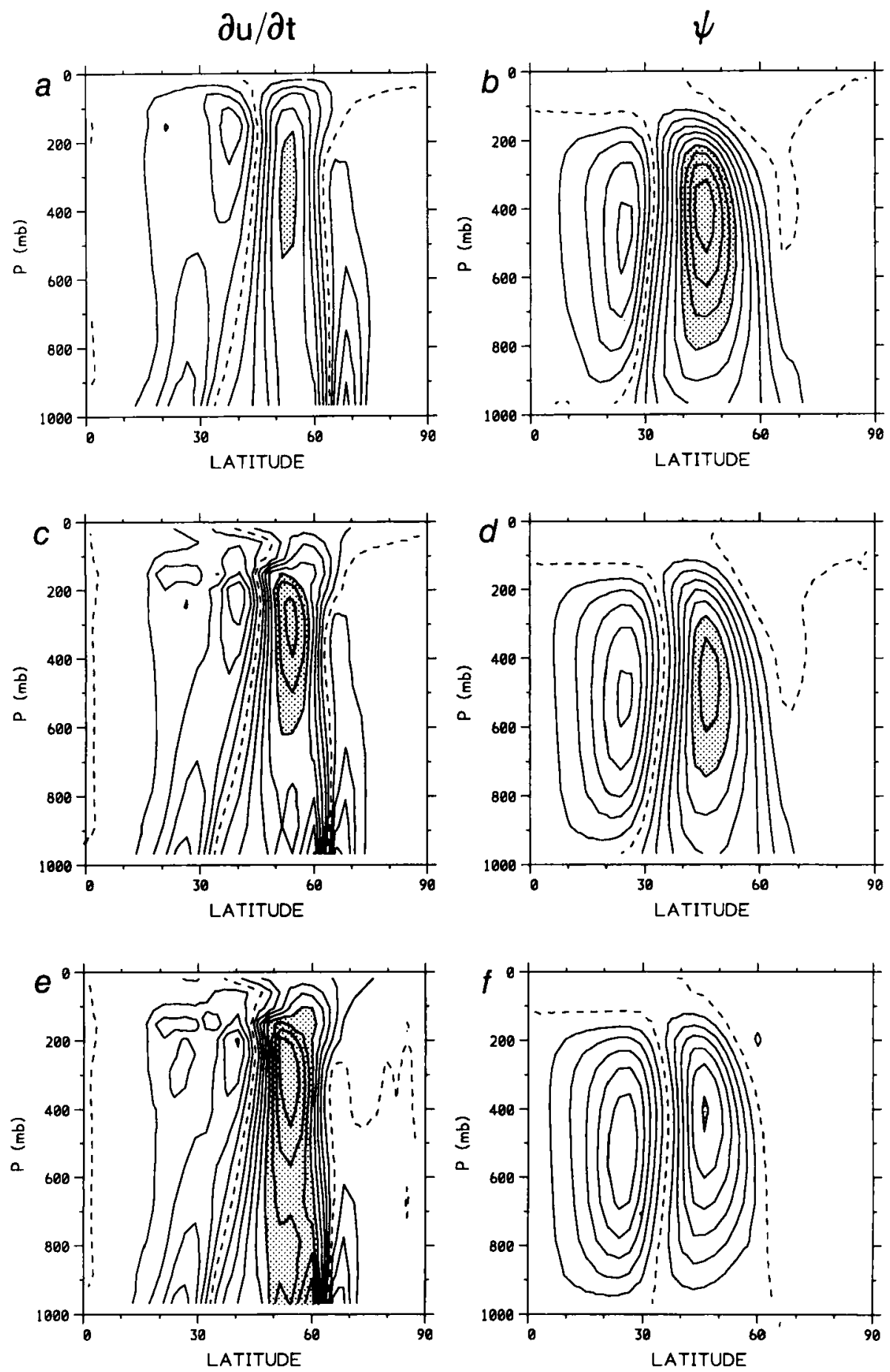

Figure 7. As in Fig. 6, but for the decay stage, days $6-12$, and with contour intervals of $10^{-5} \mathrm{~m} \mathrm{~s}^{-2}$ for $\partial u / \partial t$ and $2 \times 10^{-2} \mathrm{~m} \mathrm{~s}^{-1} \times 1000 \mathrm{mb}$ for $\psi$. Stippled regions represent $\partial u / \partial t$ greater than $5 \times 10^{-5} \mathrm{~m} \mathrm{~s}^{-2}$ and $\psi$ less than $-1.2 \times 10^{-1} \mathrm{~m} \mathrm{~s}^{-1} \times 1000 \mathrm{mb}$. 

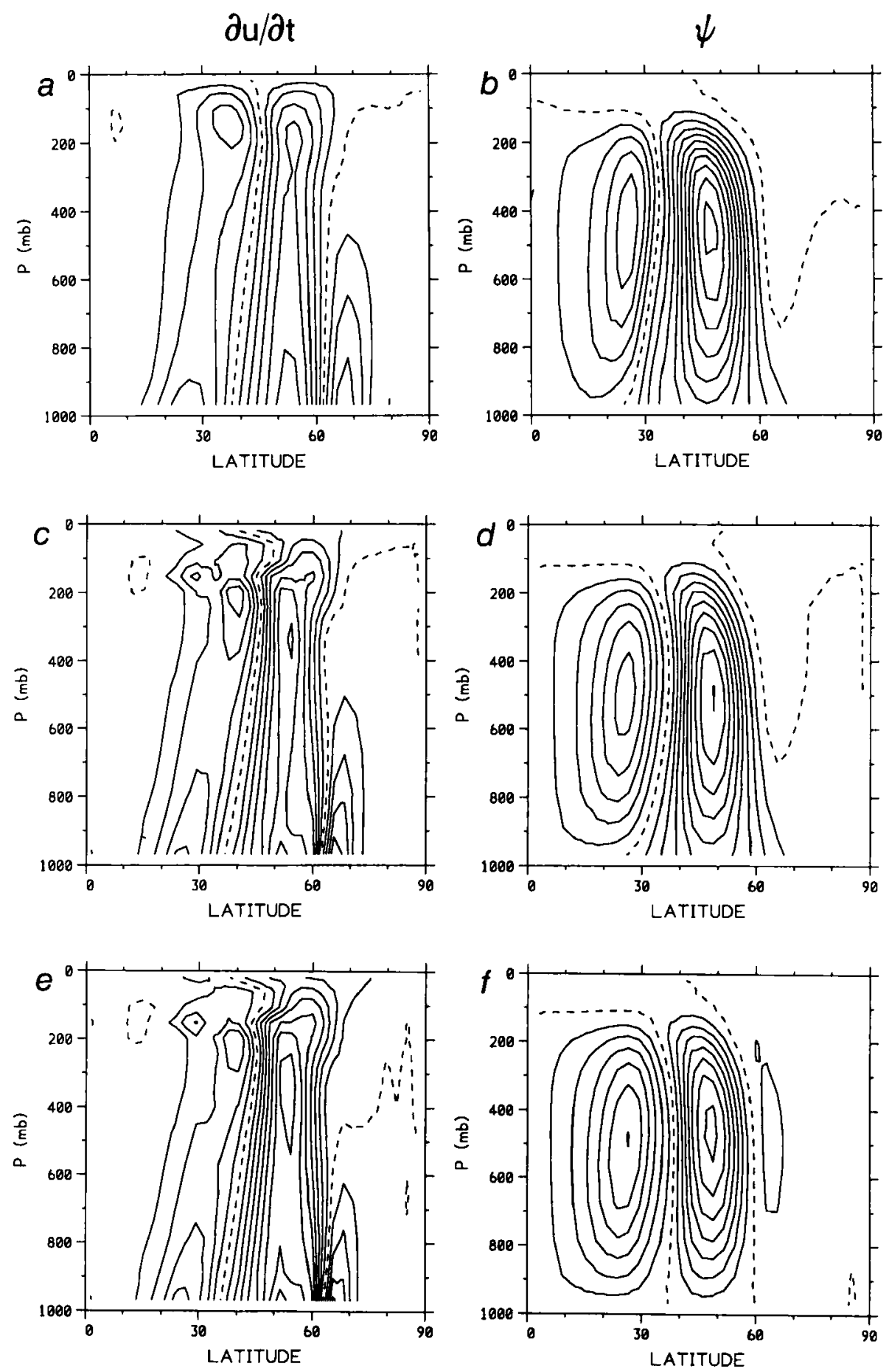

Figure 8. As in Fig. 6, but for the complete life-cycle, days 0-15 (no stippling). 
less close here than in Fig. 6; the inversion again overestimates the strength of the response, more so without the surface pressure effect than with it, but the structure of the response is also more different. The principal maximum, at $300 \mathrm{mb}$ and $55^{\circ} \mathrm{N}$, is too large by about $35 \%$ with the surface pressure effect, and by about $65 \%$ without it. The meridional circulation, on the other hand, is remarkably good (particularly the one with the surface pressure effect, of course), although, as in Fig. 6, the strength of the tropical cell is relatively exaggerated in the inversion. The importance of the surface pressure effect (as reflected in the difference in magnitude between Figs. 7(c) and (e), and in the number of contours intersecting the ground in Fig. $7(\mathrm{~d})$ ) is perhaps surprising, at first sight, given the fact that the forcing is concentrated at tropopause level (Figs. 5(d), (f)), but is understandable in view of the results presented in section 3.

For completeness, the same set of diagnostics is presented for the full run, days $0-15$, in Fig. 8. The picture is rather similar to that from days $6-12$, and so no further comments are necessary.

It has been argued above that inclusion of the surface pressure effect is essential in order to predict the mean-flow response to a baroclinic life-cycle with any accuracy. On the other hand, the discrepancies between Figs. 8(a) and (c), for example, show that the effects considered thus far are not sufficient to obtain complete agreement. There are potentially many reasons for this. Some involve the mean flow itself, such as the fact that it may not be in a state of exact balance, and-perhaps more importantly-that it is not the state of rest that has been assumed in the preceding inversion problem. Another is that the eddy forcings (4.1), (4.2) assume quasi-geostrophic scaling for the eddies, which is not necessarily valid.

The latter assumption may be tested rather easily, by using the full expressions for the eddy flux convergences

$$
\begin{gathered}
F=-a^{-1}\left(1-\mu^{2}\right)^{-1 / 2} \frac{\partial}{\partial \mu}\left\{\left(1-\mu^{2}\right) \overline{u^{\prime} v^{\prime}}\right\}-\frac{\partial}{\partial p}\left(\overline{u^{\prime} \omega^{\prime}}\right) \\
Q=-a^{-1} \frac{\partial}{\partial \mu}\left\{\left(1-\mu^{2}\right)^{1 / 2} \overline{v^{\prime} T^{\prime}}\right\}-\frac{\partial}{\partial p}\left(\overline{\omega^{\prime} T^{\prime}}\right) .
\end{gathered}
$$

These have been evaluated from the life-cycle simulation, and are shown in Fig. 9 for the periods 0-6 days and 6-12 days. Comparison with Fig. 5 shows that the extra (ageostrophic) terms make a considerable difference to the eddy forcing, particularly to the thermal forcing $Q$, where they add a substantial low-level cooling during both periods. The mechanical forcing $F$ is also noticeably changed in the period 6-12 days, where the extra terms are associated with shallow westerly forcing aloft and a deeper, but weaker, easterly forcing below.

The response to the modified forcing over the period 0-6 days, as predicted by the inversions with the two different lower boundary conditions, is shown in Fig. 10. The most striking change in the mean-flow response is the improvement in the prediction of the strength of the equatorward meridional cell. This occurs almost entirely as a result of the changed thermal forcing, and experiments in which the extra terms in the thermal forcing were used as the sole forcing showed that in the associated adjustment the surface pressure change was very small. It is therefore not surprising that the improvement is seen with both boundary conditions, although, as expected, the predicted circulation with the correct lower boundary condition is closer to that in Fig. 6(b). There is little change in the low-level acceleration as a result of the extra forcing terms; at upper levels the negative-positive pattern is enhanced. This leaves the predicted negative acceleration too strong, but the predicted positive acceleration much improved. 
Days 0-6
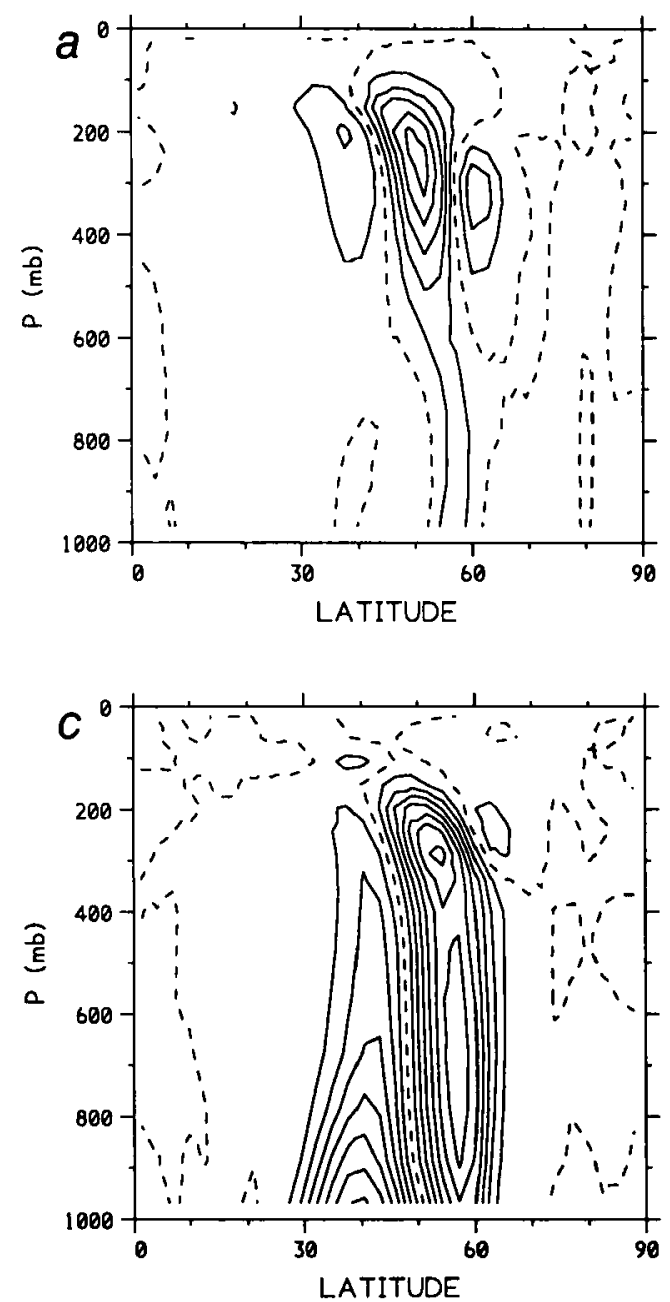

Days 6-12
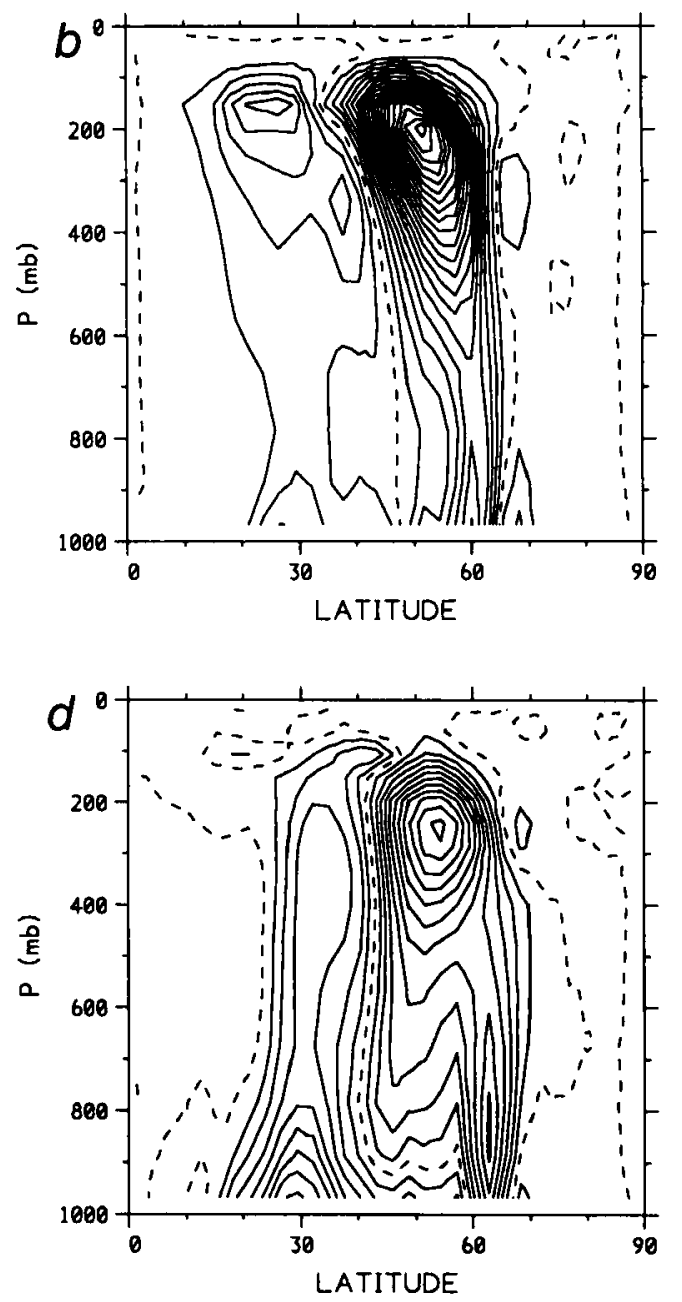

Figure 9. Full ageostrophic forcing terms produced by the life-cycle simulation for the periods $0-6$ and $6-12$ days. (a), (b) Mechanical forcing $F$ as defined by (4.3), contour interval $10^{-5} \mathrm{~m} \mathrm{~s}^{-2}$. (c), (d): Thermal forcing $Q$ as defined by (4.4), contour interval $10^{-5} \mathrm{~K} \mathrm{~s}^{-1}$. The zero contours are dashed. Compare Fig. 5 .

In the period 6-12 days, for which the response is shown in Fig. 11, it is more difficult to identify any specific improvements in $\partial u / \partial t$ and $\psi$. The overprediction of upper-level accelerations (of both signs) seen in Fig. 7 has, if anything, been made worse by including the ageostrophic terms. Examination of the response to the extra terms alone shows that, as for the growth period, the response to the extra thermal forcing is not associated with a large surface pressure change, and is therefore insensitive to the lower boundary condition. On the other hand the response to the extra mechanical forcing is sensitive to the boundary condition, since the extra forcing is relatively broad, and the associated accelerations are larger with the $\omega=0$ boundary condition. However, when included in the response to the forcing as a whole, as is the case in Fig. 11, these differences are virtually undetectable.

There is a much more obvious improvement in the temperature tendency, $\partial T / \partial t$, as 
a result of including the ageostrophic terms. This is evident from Fig. 12, which shows the time-averaged $\partial T / \partial t$ in the life-cycle, and as predicted by the inversion (with the correct lower boundary condition) with both geostrophic and full, ageostrophic expressions for the eddy fluxes, for the periods $0-6$ days and 6-12 days. Not least, the improvement gives confidence that the ageostrophic terms have been included correctly, and that they are vital to the improvement of the inversion as a whole. The continuing disagreement between simulated and predicted upper-level accelerations may well be an indication that the non-resting basic state must be accounted for to obtain further improvement.
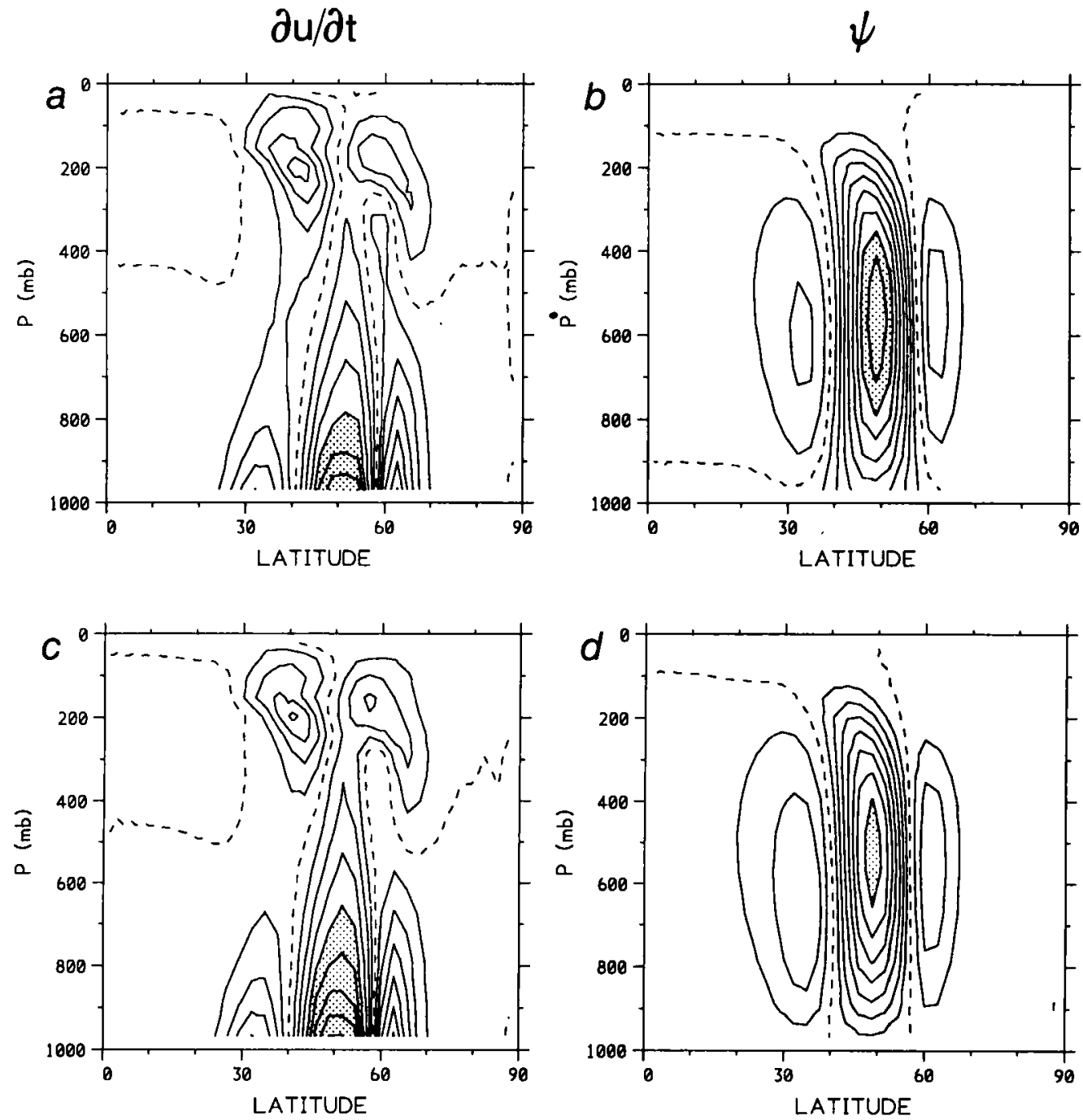

Figure 10. Predicted response to a baroclinic life-cycle during the growth stage, days $0-6$, using the full expressions for mechanical and thermal forcing, showing zonal wind acceleration $\partial u / \partial t$ and meridional streamfunction $\psi$. (a), (b): Correct lower boundary condition. (c), (d): $\omega=0$ at lower boundary. Contour intervals and stippling as in Fig. 6. 

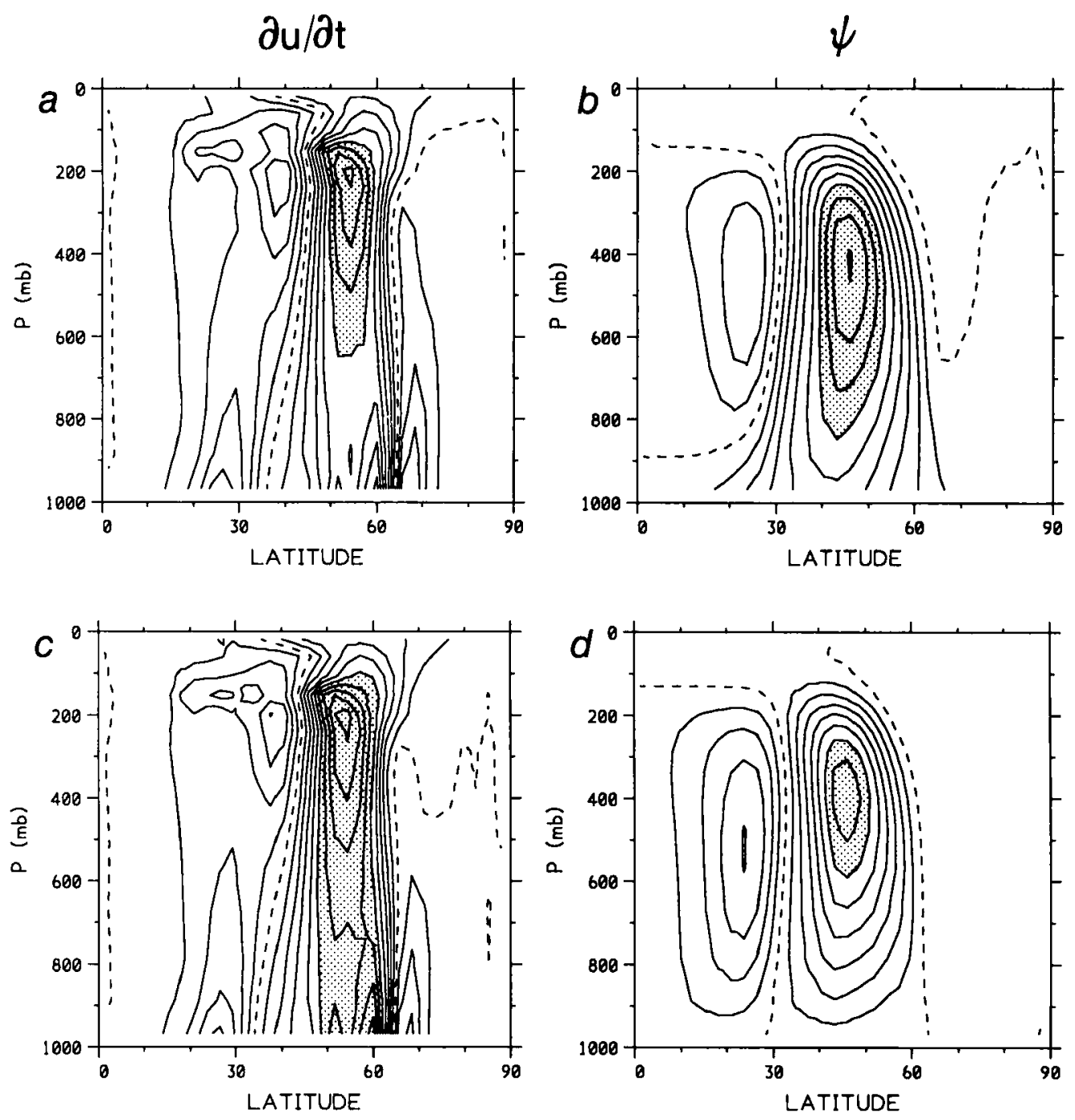

Figure 11. As Fig. 10, but for the decay stage, days 6-12. Contour intervals and stippling as in Fig. 7.

\section{Discussion}

In quasi-geostrophic theory, it is commonplace to impose the lower boundary condition that, in the absence of topography, $D p / D t \equiv \omega=0$ on the lowest pressure surface $p=p_{\mathrm{o}}$ (e.g. Pedlosky 1979, $\S 6.3$; Gill 1982, p.185). This is an approximation to the correct condition of no flow across the lower boundary. The validity of this approximation does not follow simply from the assumption of small Rossby number, but requires the additional assumption (2.18)-a condition which depends on the horizontal scale of the motion, and which is certainly questionable for planetary scales. The importance of using the correct lower boundary condition in studies of free long waves is widely appreciated, and has been highlighted by Wiin-Nielsen (1971) and White (1978), among others. 
Days 0-6
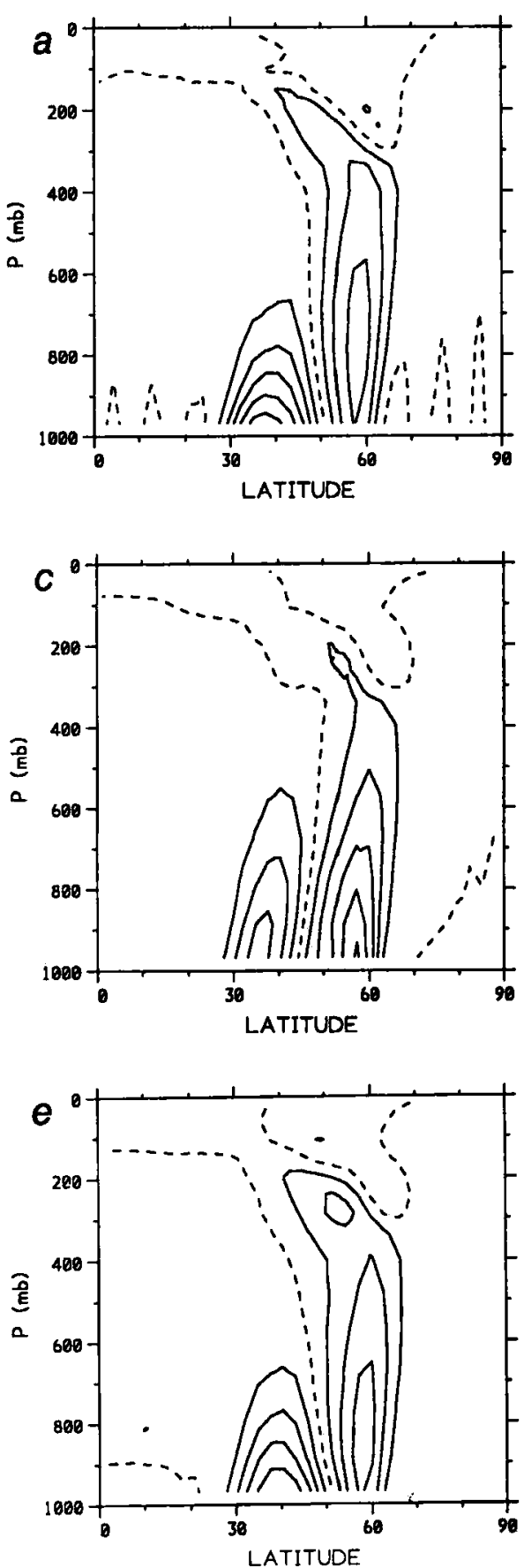

Days 6-12
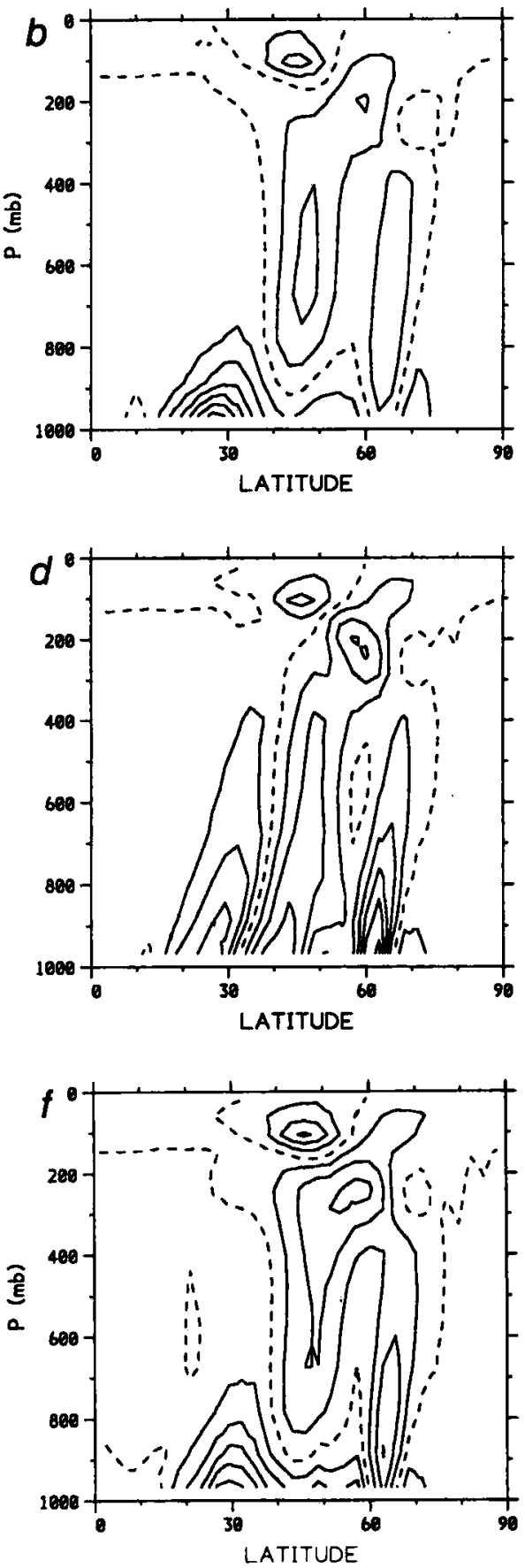

Figure 12. Temperature tendency $\partial T / \partial t$ in the model (a) averaged over $0-6$ days and (b) over $6-12$ days; as predicted by the inversion with geostrophic eddy fluxes (c) $0-6$ days and (d) 6-12 days; and as predicted by the inversion with full ageostrophic eddy fluxes (e) 0-6 days and (f) 6-12 days. Contour interval $10^{-5} \mathrm{~K} \mathrm{~s}^{-1}$. 
In this paper the question of the lower boundary condition has been addressed within the context of the classical problem of the response of a balanced vortex to zonallysymmetric forcing. Starting with Eliassen (1951), this problem has traditionally been solved in pressure or log-pressure coordinates, subject to the approximate condition $\omega=0$ at $p=p_{0}$, and therefore assuming that the mass in each vertical column remains constant. Use of the correct lower boundary condition removes this constraint, and explicitly allows a meridional redistribution of mass as part of the response. In contrast to the classical response problem, therefore, part of the applied torque may appear as a change in the planetary angular momentum. The results presented in section 3 show that this fraction may be quite considerable: one half, for example, for mid-tropospheric mechanical forcing of the planetary-scale $n=2$ Hough mode (Table 2). The response in the relative angular momentum is correspondingly weaker. Because the size of the difference increases downwards (Fig. 3), the vertical structure of the response is also affected (see Fig. 2 and Table 2).

As the horizontal scale decreases, so too does the sensitivity of the response to the lower boundary condition. The response as a whole also becomes more strongly confined in the vertical, which means that the surface pressure effect can be significant only for low-level forcing. But for planetary-scale forcing, the vertical confinement is so weak that the response is strongly affected even for forcing at tropopause level. These features may be clearly seen in the vertical profiles of $\partial u / \partial t$ shown in Fig. 4 .

The significance of these effects in a realistic context has been illustrated by studying the zonal-mean response during a baroclinic life-cycle in a primitive-equation model (section 4). There is, in fact, a significant meridional redistribution of mass (and concomitant change in the surface pressure distribution) during the course of the life-cycle. Solution of the classical quasi-geostrophic response problem, with the surface pressure tendency neglected, overpredicts the zonal wind acceleration for the complete life-cycle by about $50-60 \%$, although the structure of the response is fairly close to that observed in the model. Use of the correct lower boundary condition reduces this difference to about $15-30 \%$.

While the surface pressure effect is evidently an important part of time-dependent problems, one might be tempted to ignore it when considering time-averaged problems such as the maintenance of the general circulation. However, it is essential to be quite clear about what one is doing when the response problem is applied in a time-averaged context. In trying to deduce the role of the eddies in the general circulation, for example, the simplest diagnostic approach is presumably that of examining the budgets implied by the time-averaged versions of (2.1)-(2.5). The difficulty with this is that it provides no causal information; rather, through the continuity equation (2.5) one simply obtains a consistency condition on the forcings $F$ and $Q$. There may be circumstances where this seems appropriate; for instance, in the middle atmosphere one might imagine the dynamical forcing associated with wave breaking as working against a 'radiative spring' (e.g. Fels 1985; Andrews 1987). In the troposphere, on the other hand, the range of mechanical and thermal forcing mechanisms is so complex (including those associated with the planetary boundary layer) that it is difficult to make any clear arguments concerning which are 'cause' and which are 'effect'.

This dilemma has encouraged a return to the time-dependent response problem in order to make causal deductions about the role of various phenomena in the maintenance oi the general circulation. In such an approach the zonal wind tendencies associated with distinct physical processes are identified by solving the inversion problem using, separately, the forcing due to each of those processes. The sum of the resulting tendencies must then be equivalent to the time-averaged zonal-mean momentum equation, viz. 


$$
\left\{\frac{\partial \bar{u}}{\partial t}\right\}_{\text {eddies }}+\left\{\frac{\partial \bar{u}}{\partial t}\right\}_{\text {friction }}+\left\{\frac{\partial \bar{u}}{\partial t}\right\}_{\text {moisture }}+\ldots \text { etc } \ldots=0
$$

for example, but it is the nature of the individual, non-zero contributions to the left-hand side which gives insight, rather than the fact that their sum must vanish. This is the philosophy adopted in studies such as those of Crawford and Sasamori (1981) and Pfeffer (1981, 1987). However, these authors have solved inversion problems in which the surface pressure tendency is neglected, which seems inconsistent insofar as surface pressure and zonal velocity should be treated on the same footing. Thus there is an equation for the surface pressure which is equivalent to (5.1), and again it is the individual contributions to the left-hand side of that equation which are of interest. As has been shown here, accounting for surface pressure changes as part of the response is not only required for reasons of consistency, but can make a substantial difference to the rest of the response. This suggests that in future such observational studies, it might be advisable to apply the correct lower boundary condition in the inversion problem.

\section{ACKNOWLEDGEMENTS}

The authors would like to thank Dr A. A. White for helpful comments concerning the wider context of the surface pressure effect, and the atmospheric dynamics group at Reading University for allowing the use of their primitive-equation model. Referees' comments were especially detailed and helpful. PHH has been supported by a Royal Society Meteorological Office Research Fellowship and by Queens' College, Cambridge. TGS has been supported by the UK Natural Environment Research Council and by St Catharine's College, Cambridge, and is currently supported by the Natural Sciences and Engineering Research Council and the Atmospheric Environment Service of Canada. The manuscript was revised while PHH was visiting the Physics Department at the University of Toronto with support from the Blythe Fund.

Andrews, D. G.

Andrews, D. G. and McIntyre, M. E.

Crawford, S. L. and Sasamori, T.

Dickinson, R. E.

1969

Eliassen, A.

1951

Fels, S. B.

Gill, A. E.

Hayashi, Y.

Hoskins, B. J.

\section{REFERENCES}

1987 The influence of atmospheric waves on the general circulation of the middle atmosphere. Phil. Trans. R. Soc. Lond., A, 323, 693-705

Planetary waves in horizontal and vertical shear: The generalized Eliassen-Palm relation and the mean zonal acceleration. J. Atmos. Sci., 33, 2031-2048

A study of the sensitivity of the winter mean meridional circulation to sources of heat and momentum. Tellus, 33, 340-350

Theory of planetary wave-zonal flow interaction. J. Atmos. Sci. , 26, 73-81

Slow thermally or frictionally controlled meridional circulation in a circular vortex. Astrophys. Norveg., 5, 19-60

1985 Radiative-dynamical interactions in the middle atmosphere. Advances in Geophysics, 28A, 277-300

1982 Atmosphere-ocean dynamics. Academic Press

1985 Theoretical interpretations of the Eliassen-Palm diagnostics of wave-mean flow interaction. Parts I and II. J. Meteorol. Soc. Jap. , 63, 497-512; 513-521

1983 Modelling of the transient eddies and their feedback on the mean flow. Pp. 169-199 in Large-scale dynamical processes in the atmosphere. Edited by B. J. Hoskins and R. P. Pearce. Academic Press 
Hoskins, B. J. and Simmons, A. J. 1975

Kuo, H.-L.

Longuet-Higgins, M. S.

Palmer, T. N., Shutts, G. J. and Swinbank, $\mathbf{R}$.

Pedlosky, J.

Pfeffer, R. L.

Phillips, N. A.

Plumb, R. A.

Simmons, A. J. and Hoskins, B. J. 1978

1980

White, A. A.

1956

1968

1986

1964

1979

1981

1987

1954

1982

1980

1977

1978

White, A. A. and Gadian, A. M.

Wiin-Nielsen, A.
A multi-layer spectral model and the semi-implicit method. $Q$. J. R. Meteorol. Soc., 101, 637-655

Forced and free meridional circulations in the atmosphere. $J$. Meteorol., 13, 561-568

The eigenfunctions of Laplace's tidal equations over a sphere. Phil. Trans. R. Soc. Lond., A, 262, 511-607

Alleviation of a systematic westerly bias in general circulation and numerical weather prediction models through an orographic gravity wave drag parametrization. $Q . J . R$. Meteorol. Soc., 112, 1001-1039

The stability of currents in the atmosphere and oceans. Part I. J. Atmos. Sci., 21, 201-219

Geophysical fluid dynamics. Springer-Verlag

Wave-mean flow interaction in the atmosphere. J. Atmos. Sci., 38, $1340-1359$

Comparison of conventional and transformed Eulerian diagnostics in the troposphere. $Q . J . R$. Meteorol. Soc., 113, 237-254

Energy transformations and meridional circulations associated with simple baroclinic waves in a two-level, quasi-geostrophic model. Tellus, 6, 273-286

Zonally symmetric Hough modes and meridional circulations in the middle atmosphere. J. Atmos. Sci., 39, 983-991

The life cycles of some nonlinear baroclinic waves. ibid., 35, $414-432$

Barotropic influences on the growth and decay of nonlinear baroclinic waves. ibid., 37, 1679-1684

Modified quasi-geostrophic equations using geometric height as vertical coordinate. Q.J.R. Meteorol. Soc., 103, 383396

A note on the horizontal boundary condition in quasi-geostrophic models. J. Atmos. Sci., 35, 735-739

1979 Baroclinic instability governed by the modified quasi-geostrophic equations. Q. J. R. Meteorol. Soc., 105, 759-766

1971 On the motion of various vertical modes of transient, very long waves. Parts I and II. Tellus, 23, 87-98; 207-217 